\title{
Abgrenzung von Arbeitsmarktregionen - ein Methodenvorschlag
}

\author{
Per Kropp · Barbara Schwengler
}

Eingegangen: 14. Juni 2010 / Angenommen: 6. Januar 2011 / Online publiziert: 15. Februar 2011

(C) Springer-Verlag 2011

Zusammenfassung Die vorliegende Studie präsentiert einen Methodenvorschlag für gemeinde- und kreisscharfe Abgrenzungen von Arbeitsmarktregionen auf der Basis von Pendlerverflechtungen. Die Abgrenzung nutzt als Verfahren eine Weiterentwicklung der graphentheoretischen Methode, deren Güte mit dem Modularitätsmaß Q aus der Netzwerkforschung gemessen wird. Die Studie basiert auf Gemeindedaten für Deutschland für die Jahre 1993 bis 2008. Als Ergebnis der Analysen werden gemeinde- und kreisscharfe Abgrenzungen mit 50 Arbeitsmarktregionen vorgestellt. Dieses Abgrenzungsergebnis liefert recht größenheterogene Regionen, besitzt aber über den betrachteten Zeitraum eine hohe zeitliche Stabilität. Es wird dargelegt, dass auch eine kreisscharfe Abgrenzungsvariante ein sehr gutes Ergebnis für die Erfassung von Pendlerverflechtungen und einen hohen Selbstversorgungsgrad der Regionen liefert. Der Vergleich mit bisherigen Abgrenzungen funktionaler Räume in Deutschland anhand verschiedener Merkmale bestätigt die Güte der gefundenen Abgrenzung.

Schlüsselwörter Abgrenzung · Arbeitsmarktregion · Pendlerverflechtung · Funktionale Region · Modularität

Elektronisches zusätzliches Material Die Online-Version dieses Artikels (doi: 10.1007/s13147-011-0076-4) enthält zusätzliches Material, welches für autorisierte Benutzer zugänglich ist.

\section{Dr. P. Kropp}

IAB Sachsen-Anhalt-Thüringen

Regionalforschung des Instituts für Arbeitsmarktund Berufsforschung in der Regionaldirektion

Sachsen-Anhalt-Thüringen der Bundesagentur für Arbeit,

Frau-von-Selmnitz-Str. 6, 06110 Halle, Deutschland

E-Mail: per.kropp@iab.de

B. Schwengler $(\square)$

Institut für Arbeitsmarkt- und Berufsforschung,

Regensburger Straße 104, 90478 Nürnberg, Deutschland

E-Mail: barbara.schwengler@iab.de

\section{Delineation of Functional Labour Market Regions-A Methodological Approach}

\begin{abstract}
This paper proposes a methodological approach for delineating functional labour market regions based on commuter flows between German municipalities for the years 1993 to 2008 using the graph theory. The modularity commonly used in the analysis of networks is a suitable indicator of measuring the quality of delineations. We present a delineation of 50 labour market regions of quite different sizes that is robust for all years in the analysis. A further delineation based on commuter flows between districts is nearly as good in measuring the employment self-containment-ratio as the delineation based on commuter flows between municipalities. Comparing the presented delineation with former delineations for Germany by different statistical measures confirms the quality of the result.
\end{abstract}

Keywords Delineation - Labour market regions · Commuter flows $\cdot$ Functional regions $\cdot$ Modularity

\section{Einleitung}

Das Ziel der vorliegenden Arbeit ist die Abgrenzung von Arbeitsmarktregionen für Deutschland auf der Basis von Pendlerverflechtungen zwischen Gemeinden. Solche funktionalen Regionen bieten den Vorteil, dass sie die räumlichen Aspekte wirtschaftlicher Aktivitäten so gut wie möglich widerspiegeln und somit relevante Analyseeinheiten für die Regionalforschung liefern. Dies können administrative Gebietseinheiten in der Regel nicht leisten, weil sie meist historisch gewachsen sind und sich an Verwaltungsstrukturen orientieren. 
Frühere Studien zur Abgrenzung von regionalen Arbeitsmärkten basierten auf verschiedenen methodischen Verfahren, wie der „Clusteranalyse“ (Tolbert/Killian 1987), der Kennziffernmethode (ONS/Coombes 1998) und der Faktorenanalyse (Eckey/Kosfeld/Türck 2006). In einer Untersuchung jüngeren Datums, bei der ausgewählte Methoden anhand diverser Gütemaße miteinander verglichen wurden, lieferten Cluster-Verfahren und die graphentheoretische Methode die besten Ergebnisse (Kropp/Schwengler 2008).

Die vorliegende Arbeit basiert auf einer Weiterentwicklung der graphentheoretischen Methode von Rabino und Occelli (1997). Zur Beurteilung der Güte dieser Abgrenzung und für den Vergleich mit anderen Abgrenzungen wird auf das in der Netzwerkforschung entwickelte Modularitätsmaß Q von Newman zurückgegriffen (Newman/Girvan 2004). Als Datengrundlage dient die Zahl der sozialversicherungspflichtig Beschäftigten in Deutschland für die Jahre 1993 bis 2008 jeweils zum 30. Juni eines jeden Jahres auf Gemeindeebene. Da die Gemeinden unterschiedlich groß sind, werden zur Homogenisierung der Ausgangseinheiten die rund 12.000 Gemeinden unter Berücksichtigung von Entfernung und Einwohnerzahl zu 2.000 Gemeinderegionen zusammengefasst. Die Nutzung der Zeitreihe erlaubt es, die Stabilität der Abgrenzungen im Zeitverlauf zu überprüfen.

Die Arbeit ist wie folgt gegliedert: Im nächsten Abschnitt wird ein Überblick über den Forschungsstand gegeben. In Abschn. 3 folgt die Beschreibung sowohl der Datenbasis als auch des Verfahrens zur Zusammenfassung der Gemeinden zu Gemeinderegionen. Im Anschluss daran werden in Abschn. 4 die graphentheoretische Methode und das hier angewandte weiterentwickelte Verfahren sowie das Modularitätsmaß Q als Gütekriterium erläutert. Das Ergebnis der analytisch besten Abgrenzung, ihre zeitliche Stabilität, gemeinde- und kreisgenaue Abgrenzungen, wichtige deskriptive Merkmale sowie ein Vergleich mit anderen Abgrenzungen werden in Abschn. 5 vorgestellt und diskutiert. Die Arbeit schließt mit Abschn. 6, der eine zusammenfassende Einschätzung der gefundenen Ergebnisse sowie einen Ausblick für weitere Forschungsvorhaben enthält.

\section{Forschungsstand}

Das Konzept funktionaler Regionen versucht die Realität räumlicher ökonomischer Prozesse so gut wie möglich abzubilden. Funktionale Regionen sind demnach definiert als Regionen, die einen Großteil der ökonomischen Aktivitäten innerhalb ihrer Grenzen konzentrieren (Smart 1974: 261; Coombes/Green/Openshaw 1986: 944; van der Laan/ Schalke 2001: 205; Bongaerts/Cörvers/Hensen 2004: 2). Häufig wird in diesem Zusammenhang von self-containment oder Selbstversorgungsgrad gesprochen.
Die Bedeutung von Agglomerationsvorteilen und -nachteilen für die Entwicklung regionaler Disparitäten wurde in der ökonomischen Theorie schon früh betont und ist in den letzten Jahren im Zusammenhang mit der Neuen Ökonomischen Geographie (Krugman 1991) wieder stärker diskutiert worden (vgl. Sternberg 2001; Wintzer 2007; aktuelle Anwendungen bei Kosfeld/Eckey/Türck 2008 oder Dauth 2010). Die Bündelung verschiedener Aktivitäten kann positive Skaleneffekte erzeugen und so durch höhere Arbeits- und Kapitalproduktivitäten zu einer höheren Wettbewerbsfähigkeit in der Region, aber durch darüber hinausgehende räumliche externe Effekte (,Spillover“-Effekte) auch in benachbarten Regionen beitragen. Dies können zum einen Lokalisationsvorteile sein, wenn sich Unternehmen in unmittelbarer Nähe zueinander ansiedeln, um so sinkende Durchschnittskosten aufgrund größerer Produktionsmengen (economies of scale) zu realisieren. Zugleich können sie von einem gemeinsamen Pool an Arbeitskräften profitieren. Zum anderen ergeben sich Urbanisationsvorteile der Regionen, wenn Unternehmen verschiedene Produkte gleichzeitig am gleichen Standort produzieren, um so Kosten $\mathrm{zu}$ sparen (economies of scope). Die Verdichtung der ökonomischen Aktivitäten zieht wiederum eine Verdichtung der Bevölkerung nach sich, so dass Unternehmen aus verschiedenen Sektoren auf ein gemeinsames Marktpotenzial an Kunden zurückgreifen können.

Die Theorie der Agglomerationsvorteile geht auf die Theorie der Zentralen Orte zurück, die schon 1933 von Walter Christaller entwickelt und von August Lösch (1940) erweitert wurde. Das auf der Theorie der Zentralen Orte aufbauende Zentrale-Orte-Konzept nahm in den 1960er und 1970er Jahren eine zentrale Rolle in der deutschen Raumordnungspolitik ein. Dieses orientierte sich zunächst an der Versorgung der Bevölkerung zur Schaffung gleichwertiger Lebensbedingungen in den Regionen und wurde dann in den einzelnen Bundesländern in unterschiedlichem Umfang um Entwicklungsfunktionen erweitert. Seit den 1980er Jahren geriet es immer mehr in die Kritik, gewann aber in den 1990er Jahren mit der Wiedervereinigung und auf europäischer Ebene wieder an Bedeutung und nimmt nach wie vor einen wichtigen Stellenwert bei der Landes- und Regionalplanung ein (Blotevogel 1996: 655; Blotevogel 2005: 1314).

Zudem stellt sich bei jeder Analyse von Daten die Frage nach der geeigneten Gebietseinheit. Die Ergebnisse von Analysen können je nach Zuschnitt der zugrundeliegenden Gebietseinheit zum Teil sehr widersprüchlich ausfallen. Vor dem Hintergrund des Problems der veränderbaren Gebietseinheit (modifiable areal unit problem (MAUP)) ist es daher von entscheidender Bedeutung, die für den jeweiligen Zweck bzw. Politikbereich der Analyse geeignete Gebietsabgrenzung zu verwenden (vgl. hierzu auch Openshaw 1984; Madelin/Grasland/Mathian et al. 2009). 
Zur Abgrenzung von funktionalen Regionen werden in der Regel Pendlerverflechtungen zwischen Wohnort und Arbeitsort analysiert. Kleinräumige administrative Regionen bilden dabei den Ausgangspunkt. Es können einseitige Pendlerströme, zweiseitige Pendlerverflechtungen oder der Zugang von Arbeitskräften zu Arbeitsplätzen bzw. der Zugang von Firmen zu Arbeitskräften Anwendung finden (Karlsson/Olsson 2006: 5 ff.). Um die Geschlossenheit einer Region zu gewährleisten, sollte der Selbstversorgungsgrad bei geschlossenen funktionalen Regionen möglichst hoch sein (Coombes/Green/Openshaw 1986: 944), was eine hohe Pendlerverflechtung innerhalb der Region und eine geringe Pendlerverflechtung zu anderen Regionen voraussetzt (Hensen/Cörvers 2003: 9). Der Grad für die Geschlossenheit dieser Arbeitsmärkte für Arbeit und Wohnen kann anhand verschiedener Kriterien gemessen werden, wie beispielsweise mit dem home-work-ratio (HWR), dem housing self-containment ratio (HSC) oder dem employment self-containment ratio (ESC) (van der Laan/Schalke 2001: 209 ff.). Die home-work-Relation (HWR) misst die Differenz von Ein- und Auspendlern im Verhältnis zu allen in einer Region wohnenden Beschäftigten, die housing self-containment-Relation (HSC) gibt an, wie viele der in einer Region arbeitenden Personen auch in dieser Region wohnen (und ist damit identisch mit dem oben beschriebenen Selbstversorgungsgrad) und die employment self-containment-Relation (ESC) ermittelt, inwiefern eine Region genug Arbeitsplätze für die dort wohnende Bevölkerung bereitstellt. Je nach Verwendungszweck können aber darüber hinaus auch funktionale Regionen über weitere Ströme, wie z. B. von Gütern und Dienstleistungen, Kommunikation oder Verkehr, oder anhand von regionalen Preisniveaus, wie z. B. Landpreisen, gebildet werden (Bode 2008: 144).

Zur Bestimmung der Grenzen von Wirtschaftsräumen gibt es eine Vielzahl von Verfahren. Einen aktuellen Überblick geben van Nuffel (2007) und Kropp/Schwengler (2008). International bekannt sind Travel-to-Work Areas (TTWA), (Local) Labour Market Areas (LLMA) oder Standard Metropolitan Statistical Areas (SMSA). Travel-to-Work Areas sind beispielsweise relevant für Arbeitsmarktanalysen und Industriepolitik, als Basis für die Statistik und auch zur Reorganisation der Regionalverwaltung in Großbritannien (ONS/Coombes 1998). Die Local Labour Market Areas finden Anwendung in den Niederlanden (van der Laan/Schalke 2001; Cörvers/Hensen/Bongaerts 2009), Belgien (van Nuffel 2007), Schweden (Karlsson/Olsson 2006), Deutschland (Eckey/Kosfeld/Türck 2006), Spanien (Casado-Díaz 2000) und Italien, während Standard Metropolitan Statistical Areas als relevante Einheiten für die Wirtschaftspolitik in den USA herangezogen werden.

Wissenschaftliche Studien zur Abgrenzung von regionalen Arbeitsmärkten gibt es für Deutschland seit Anfang der 1970er Jahre (Klemmer/Kraemer 1975; Eckey 1988;
Eckey/Klemmer 1991; Budde/Eckey/Klemmer 1993; Budde/Eckey/Schrumpf 1995; Eckey 2001; Eckey/Kosfeld/ Türck 2006). Regionale Untersuchungen jüngeren Datums existieren für die Bundesländer Bremen (Kropp 2009), Thüringen und Sachsen-Anhalt (Granato/Farhauer 2007) sowie Berlin und Brandenburg (Binder/Schwengler 2006). Eine vergleichende Studie (Kropp/Schwengler 2008) zeigt, dass graphentheoretische und Cluster-analytische Verfahren am besten geeignet sind, Pendlerverflechtungen in Arbeitsmarktregionen zu erfassen, und dass zweiseitige Pendlerströme - die Berücksichtigung von Aus- und Einpendlern zur Bestimmung der Stärke der Verflechtung zweier Regionen - die optimale Datengrundlage bieten.

Neben regionalökonomischen Analysen im theoretischwissenschaftlichen Bereich werden funktionale Gliederungen häufig auch im politisch-administrativen Bereich genutzt. Sinnvoll sind auf Pendlerströmen aufbauende Abgrenzungen von Arbeitsmarktregionen immer dann, wenn die separate Betrachtung von Wohn- und Arbeitsorten zu verzerrten Ergebnissen führt. Sollen z. B. Indikatoren miteinander verrechnet werden, die zum einen am Wohnort gemessen werden (wie z. B. die Arbeitslosenquote; vgl. bereits Smart 1974: 252 ff.) und zum anderen am Arbeitsort erfasst werden (z. B. das Einkommen), kann dies nur auf Basis einer geeigneten Gebietseinheit erfolgen, die sowohl den Wohnort als auch den Arbeitsort umfasst. Dieses Problem trifft in besonderer Weise die Stadtstaaten, wo das Einkommen der pendelnden Beschäftigten in vielen Fällen in einem Bundesland erwirtschaftet, aber in einem anderen versteuert wird. Auch die Entscheidung für die Ansiedelung von Unternehmen bzw. für die Errichtung von Infrastruktureinrichtungen sind die Pendlereinzugsbereiche von entscheidender finanzieller Bedeutung. Aktuell dienen in Deutschland die 270 Arbeitsmarktregionen der Gemeinschaftsaufgabe „Verbesserung der regionalen Wirtschaftsstruktur" als Diagnoseeinheit für die Identifikation von deutschen Fördergebieten und zum anderen die 96 Raumordnungsregionen ${ }^{1}$ für die Raumordnungsberichterstattung des Bundes. Die unterschiedlichen Abgrenzungen dieser in der Praxis verwendeten funktionalen Regionen unterliegen dabei häufig bestimmten Zwängen und Vorgaben, wie z. B. einem kreis- oder bundeslandscharfen Zuschnitt, und unterscheiden sich daher von rein wissenschaftlichen Abgrenzungen, die solche Einschränkungen nicht berücksichtigen müssen.

In jüngster Zeit finden auch Metropolregionen immer mehr Beachtung in der politischen und öffentlichen Diskussion (vgl. z. B. BBSR 2009). Metropolregionen stellen

\footnotetext{
${ }^{1}$ Die seit 1996 gültige Abgrenzung von 97 Raumordnungsregionen wurde nach den Kreisneugliederungen in Sachsen-Anhalt 2007 und Sachsen 2008 überprüft und auf nunmehr 96 Raumordnungsregionen angepasst (BBSR 2010).
} 
jedoch keine neuen Verwaltungseinheiten dar und sind auch nicht identisch mit den funktionalen räumlichen Verflechtungen, sondern sie bilden einen gemeinsamen Bezugsrahmen für gemeinsame Regionalprojekte und großräumige Partnerschaften und Kooperationen. Sie konzentrieren Bevölkerung, wirtschaftliche, politische und kulturelle Aktivitäten und lassen sich nach ihrer analytischen und normativen Bedeutung unterscheiden (ARL 2007: 3).

\section{Daten}

Die Abgrenzung von Arbeitsmarktregionen, sowohl in Deutschland als auch auf internationaler Ebene, basiert größtenteils auf Analysen von Pendlerverflechtungen. In der vorliegenden Studie wird als Datengrundlage die Zahl der sozialversicherungspflichtig Beschäftigten aus der Beschäftigtenstatistik der Bundesagentur für Arbeit für die Jahre 1993 bis 2008 jeweils zum 30. Juni eines jeden Jahres herangezogen. Das Jahr 1993 wurde als Startjahr gewählt, weil ab diesem Zeitpunkt valide Daten für ganz Deutschland vorliegen. Ergänzend zur Analyse der Pendlerverflechtungen in den einzelnen Jahren wurden für einen zusätzlichen Vergleich über veränderte Pendlerströme im Zeitablauf die ersten und letzten drei Jahre zu eigenen Kategorien zusammengefasst und separat untersucht. Dadurch lassen sich in der Regel zuverlässigere Ergebnisse erzielen als mit einzelnen Jahren (vgl. van der Laan/Schalke 2001: 206). Die Jahre 1993 bis 1995 bildeten die Kategorie ALT und die Jahre 2006 bis 2008 die Kategorie NEU. Um insbesondere die aktuellen Pendlerverflechtungen stärker zu berücksichtigen als die der vorangegangenen Jahre, erhielt in der Kategorie NEU das aktuelle Jahr 2008 ein doppelt so hohes Gewicht wie das Jahr 2007 und entsprechend das Jahr 2007 ein doppelt so hohes Gewicht wie das Jahr 2006. In analoger Weise wurden in der Kategorie ALT die Daten von 1993 am stärksten gewichtet. Die Gewichtung erfolgte, da ein besonderes Auswertungsinteresse an den ältesten und aktuellsten Daten bestand.

Die Datenquelle erfasste im Jahr 2008 insgesamt 27,3 Mio. Beschäftigte bzw. $68 \%$ aller Erwerbstätigen (Bundesagentur für Arbeit 2008: 19). Da über die Arbeitgebermeldungen an die Sozialversicherungsträger sowohl der Wohnort der Beschäftigten als auch Firmensitz bekannt ist, liegen die Daten bis auf Gemeindeebene vor. Die Pendlerverflechtungen lassen sich somit gemeindescharf abbilden.

Die Verwendung von Gemeindedaten hat gegenüber der Kreisebene den Vorteil, dass eine feinere und genauere Abgrenzung möglich ist, auch unabhängig von Kreisgebietsreformen, die beispielsweise im Jahr 2007 in SachsenAnhalt und im Jahr 2008 in Sachsen stattgefunden haben, und die zu Überschneidungen mit früheren Kreisgrenzen führten. Dem stehen als Nachteile der deutlich erhöhte
Rechenaufwand und die eingeschränkte Verfügbarkeit der genutzten Daten auf Gemeindeebene gegenüber. Es ist jedoch möglich, auf der Grundlage von Abgrenzungen auf Gemeindeebene Abgrenzungen auf Kreis- oder für andere gemeindescharfe Aggregationsebenen vorzunehmen.

Die Gemeindegrößen unterscheiden sich zwischen den Bundesländern in Deutschland z. T. erheblich. Während Gemeinden in Rheinland-Pfalz und in Schleswig-Holstein oft sehr klein sind, sind die Gemeinden in ähnlich dicht besiedelten Gebieten von Nordrhein-Westfalen um ein Vielfaches größer. Um für die Analysen eine homogenere Datengrundlage zu schaffen, wurden die rund 12.000 Gemeinden zu 2.000 Gemeinderegionen aggregiert, indem nahe beieinander liegende Gemeinden mit wenigen Einwohnern zusammengefasst wurden. Notwendig hierfür waren zunächst die Ermittlung der Distanzen zwischen allen Gemeindepaaren $\mathrm{G}_{\mathrm{ij}}$ und die Berechnung des Fusionskoeffizienten $\mathrm{F}_{\mathrm{ij}}$ nach folgender Formel:

$$
\begin{aligned}
F_{i j}= & \text { Entfernung }_{i j}^{2} \cdot\left(\text { Einwohner }_{i}+\text { Einwohner }_{j}\right) \\
& \text { mit } \mathrm{i}=1, \ldots, \mathrm{n} \text { und } \mathrm{j}=1, \ldots, \mathrm{n}
\end{aligned}
$$

Die beiden Regionen mit dem niedrigsten Koeffizienten $F_{i j}$ fusionierten zu einer Region und bekamen sowohl die Summe der Einwohner beider Ursprungsregionen als auch die Koordinaten der einwohnerreicheren Ursprungsgemeinde zugewiesen. Dieser Vorgang wurde so lange wiederholt, bis alle Regionen fusioniert waren. Bis zum Erreichen der Lösung mit 2.000 Gemeinderegionen waren insgesamt 10.224 Iterationen erforderlich. Die 2.000-Regionen-Agrenzung hat sich als günstig erwiesen, um Größenhomogenität unter den kleineren Gemeinden zu erzeugen. Bei deutlich mehr Fusionsschritten wären bereits größere Gemeinden bzw. Kleinstädte betroffen gewesen. Bei der vorliegenden Abgrenzung fanden keine Fusionen von Gemeinden mit einer Entfernung von mehr als $17 \mathrm{~km}$ (Mittelwert $4 \mathrm{~km}$ ) und einer Größe von 173.000 Einwohnern (Summe der Einwohner beider Gemeinden) statt (Mittelwert 9.747 Einwohner). Mit diesem Vorgehen ließen sich die Regionsgrößen zwischen den Bundesländern deutlich homogenisieren. In die Analyse fließen insgesamt $3.998 .000(2.000 \times 2.000-2.000)$ mögliche Pendlerbeziehungen ein. Die größten Pendlerverflechtungen bestehen zwischen benachbarten Regionen oder zu bzw. zwischen den großen Arbeitsmarktzentren.

\section{Methode}

Wie oben erwähnt, eignen sich vor allem Pendlerverflechtungen als Grundlage für die Abgrenzung von Arbeitsmarktregionen. Für die Wahl der Abgrenzungsmethode gibt es allerdings wenige theoretische Argumente (vgl. für eine solche Argumentation Eckey/Kosfeld/Türck 2006: 301 f.). 
Gegen Schwellenwertmodelle spricht in der Regel die willkürliche Auswahl des Schwellenwertes. Problematisch an hierarchischen Cluster-Verfahren ist, dass einmal vorgenommene Zuweisungen zu einem Cluster nicht veränderbar sind, auch wenn sich die Struktur der Cluster ändert. Dies ist z. B. der Fall, wenn eine Region früh im Cluster-Prozess einer Arbeitsmarktregion zugewiesen wurde, letztendlich aber stärkere Pendlerverflechtungen zu einer erst später entstandenen Arbeitsmarktregion hat. Schließlich kritisieren Eckey/Kosfeld/Türck (2006: 302) die Tatsache, dass bei den meisten Verfahren indirekte Pendlerverflechtungen nicht berücksichtigt werden. Darum ist das von ihnen vorgeschlagene faktorenanalytische Herangehen, das Regionen mit ähnlichen Pendlerstrukturen zusammenfasst, aus theoretischer Sicht zu bevorzugen. Bei einem Vergleich unterschiedlicher Methoden anhand diverser Gütemaße, wie z. B. des Selbstversorgungsgrades, der Pendlerquoten oder der Modularität, lieferten allerdings die Graphentheorie und speziell entwickelte Cluster-Verfahren die besten Ergebnisse (Kropp/Schwengler 2008: 44 ff. und 50). Nach weiteren Verbesserungen und Anpassungen des Algorithmus erwies sich das graphentheoretische Verfahren als das beste und wird darum in der vorliegenden Arbeit angewandt.

Die Graphentheorie bzw. das Konzept der dominanten Flüsse (Nystuen/Dacey 1961) und darauf aufbauende Ansätze finden bereits seit einigen Jahren auch in den Regionalwissenschaften bei der Analyse von Stromdaten und der Klassifizierung von Einzugsgebieten einzelner Ströme Anwendung (Rabino/Occelli 1997; Haag/Binder 2001; Gorman/Patuelli/Reggiani et al. 2007). Das Konzept der dominanten Flüsse geht von einer Anzahl von Knoten, den regionalen Einheiten, aus, die durch unterschiedlich starke $\mathrm{Zu}$ - und Abflüsse miteinander in Beziehung stehen. Jeder Knoten ist mit einer Hauptvernetzung, dem dominanten Strom, mit je einem weiteren Knoten verbunden. Die Zielregion jedes maximalen Stroms wird der jeweils höheren Hierarchieebene zugerechnet, wobei nur dominante Ströme von kleineren in größere (z. B. einwohnerreichere) Regionen berücksichtigt werden. So lässt sich die räumliche Struktur anhand eines hierarchisch geordneten Graphen in Form eines Baumes darstellen, dessen „Stamm“ in der Region mündet, die selbst keine Hauptverflechtung in eine größere Region ausweist, und die z. B. als Zentrum einer Arbeitsmarktregion angesehen werden kann. In diesem Konzept werden weitere Ströme neben den dominanten Strömen vernachlässigt, auch wenn diese mitunter ziemlich stark ausfallen können. Nystuen und Dacey (1961) wendeten das Verfahren zur Ordnung und Gruppierung von Städten anhand der Größe und Richtung von Ferngesprächen in ausgewählten Staaten in den USA und Kanada an. Der Ansatz lässt sich jedoch problemlos auf alle Arten von Güter- und Personenströme übertragen.
Die vorliegende Untersuchung modifiziert das graphentheoretische Verfahren. Es betrachtet nicht nur einen regionalen Teilraum, sondern das gesamte Bundesgebiet. Ausgangsbasis hierfür sind die 2.000 Gemeinderegionen und alle zwischen diesen Gemeinderegionen bestehenden aggregierten Ein- und Auspendlerströme, deren Anteil an allen in einer Gemeinderegion wohnenden Beschäftigten berechnet wird. Der größte Anteilswert bestimmt die Hauptvernetzung. Die Hauptvernetzungen, die über einem bestimmten Schwellenwert liegen und in eine größere Gemeinderegion (gemessen an der Beschäftigtenzahl am Arbeitsort) führen, bilden den Baumgraphen - die (vorläufige) Arbeitsmarktregion. ${ }^{2}$ LiegtderAnteilswert unter dem Schwellenwert, so werden die Gemeinderegionen keinem Teilgraphen bzw. keiner Arbeitsmarktregion zugewiesen. Das graphentheoretische Verfahren wird in der vorliegenden Arbeit auch dadurch modifiziert, dass die Pendlerströme zwischen den nunmehr vorhandenen Regionen (restliche Gemeinderegionen und vorläufige Arbeitsmarktregionen) neu berechnet und das beschriebene Verfahren erneut angewandt wird, bis sich keine Änderungen mehr ergeben. Dabei werden weitere Gemeinderegionen einer Arbeitsmarktregion zugewiesen und zahlreiche der vorläufigen Arbeitsmarktregionen weiter fusioniert.

Das beschriebene Verfahren kombiniert den graphentheoretischen Ansatz mit der in der Regionalforschung zur Abgrenzung von Regionen gut etablierten Schwellenwertmethode und einem hierarchischen Cluster-Verfahren. Weil dabei nicht mehr nur die Ströme zwischen einzelnen Regionen, sondern zwischen einzelnen Regionen und Agglomerationen oder zwischen Agglomerationen betrachtet werden, finden in gewisser Weise auch indirekte Pendlerverflechtungen Berücksichtigung.

Abhängig von den konkreten Schwellenwerten für den Anteil der Pendler an den in derselben Region wohnenden Beschäftigten und von der Anzahl der Wiederholungen ergeben sich unterschiedliche Abgrenzungen von Arbeitsmarktregionen, aus denen nun die ,beste ‘ ausgewählt werden muss. Als aussagekräftiges Gütekriterium zur Einschätzung, ob eine Abgrenzung von Arbeitsmarktregionen die tatsächlichen Pendlerbewegungen gut ,einfängt ${ }^{\star}$, hat sich der Modularitätsansatz von Newman (Newman/Girvan 2004) erwiesen. Dieser Ansatz hat in den letzten Jahren eine große Popularität in Disziplinen erlangt, die sich mit der Identifizierung von Gruppierungen in Netzwerken beschäf-

\footnotetext{
${ }^{2}$ Darin ähnelt das hier angewandte Verfahren dem Vorgehen von Rabino und Occelli (1997). Sie kombinierten den graphentheoretischen Ansatz mit dem Schwellenwertverfahren, indem sie Schwellenwerte für die Mindestanzahl der dominanten Pendlerströme und die Mindestgröße der Bassins (gemessen in Einwohnern und Anzahl der Gemeinden) nutzen. Baumgraphen werden nur durch Pendlerströme gebildet, die einen bestimmten Schwellenwert überschreiten. Auch Haag und Binder (2001) verwendeten dieses erweiterte Konzept für räumliche Analysen um Stuttgart und Turin.
} 
tigen (Brandes/Delling/Gaertler et al. 2008). Die Grundidee besteht darin zu messen, um wie viel besser eine Abgrenzungsmethode bei der Zusammenfassung von Einheiten zu Clustern ist als eine zufällig generierte Einteilung zu derselben Anzahl von Gruppen bzw. Arbeitsmarktregionen mit einer identischen Größenstruktur. Der empirisch ermittelten Cluster-Lösung wird also ein Nullmodell gegenübergestellt, in dem wichtige Struktureigenschaften der Verflechtungsmatrix berücksichtigt werden (die Zeilen- bzw. Spaltensummen), aber die konkrete Verteilung einer Zufallsfunktion folgt (Fortunato 2010: 86). Von einer Clusterung kann man demnach dann sprechen, wenn die beobachtete Anzahl der Kanten (im vorliegenden Fall die Anzahl der Beschäftigten) in einem Subgraphen (dem Cluster bzw. der Arbeitsmarktregion) größer ist als die Zahl der Kanten im Nullmodell, und entsprechend die Zahl der Kanten zwischen den Subgraphen geringer ist als im Nullmodell. Die Berechnung basiert auf einer symmetrischen Matrix, die als Anteilswerte an allen Einheiten auf der Diagonale die in den Clustern enthaltenen Einheiten zusammenfasst und außerhalb der Diagonale die Verflechtungen zwischen den Clustern enthält. Die Differenzen der Werte in den Clustern $i\left(e_{i i}\right)$ (Diagonalwerte) zum Quadrat der Summe der Werte in den Spalten (oder Zeilen) $a_{i}=\sum_{j} e_{i j}$ werden dann zur Modularität $Q=\sum_{i}\left(e_{i i}-a_{i}^{2}\right)$ aufsummiert. Wenn die Einteilung in Cluster nicht besser als eine Zufallseinteilung ist, nimmt Q den Wert 0 an. Werte, die sich dem Maximum von 1 nähern, weisen auf eine stark modulare Struktur hin, die durch das Clusterverfahren korrekt erfasst wurde. Üblich sind Werte zwischen 0,3 und 0,7. Beim Vergleich der verschiedenen Verfahren konnten bereits Ergebnisse mit Werten über 0,8 erreicht werden, wie sie auch der folgende Abgrenzungsvorschlag zeigt (Kropp/Schwengler 2008: 40 f.). Die Verwendung der Modularitätswerte als Gütekriterium begegnet einem Kritikpunkt an der Verwendung von Schwellenwerten, denn diese werden nicht, willkürlich' gesetzt, sondern empirisch anhand eines Qualitätskriteriums bestimmt.

Nachdem mit dem modifizierten graphentheoretischen Ansatz Abgrenzungsvarianten bestimmt und mit Hilfe der Modularitätswerte eine optimale Abgrenzung ausgewählt wurde, wird in einem abschließenden Optimierungsprozess das Ergebnis weiter verbessert. Wie bei allen hierarchischen Verfahren kommt es auch bei der hier verwendeten Methode dazu, dass Regionen frühzeitig einem Cluster zugeordnet werden, obwohl sie stärkere Pendlerverflechtungen zu erst später gebildeten Clustern haben (vgl. Eckey/Kosfeld/Türck 2006: 302). Darum werden diese Zuordnungen abschließend geprüft und gegebenenfalls optimiert. Weil die Änderung für eine Gemeinderegion auch die Zuweisung benachbarter Gemeinderegionen betreffen kann, wird der Korrekturprozess solange wiederholt, bis eine stabile Lösung gefunden ist. Dieses Verfahren wurde in Kropp (2009) beschrieben und eignet sich auch zur Anpassung der aktuellen Abgrenzung an andere Gebietsstände, z. B. für eine gemeindegenaue Abgrenzung, wie sie im nächsten Abschnitt vorgenommen wird, oder zur Anpassung an veränderte Gebietsstände infolge von Gemeinde- oder Kreisreformen.

\section{Ergebnisse}

\subsection{Die analytisch beste Abgrenzung}

Je nach Schwellenwert für den Anteil der Pendler an den in derselben Region wohnenden Beschäftigten und der Anzahl der Wiederholungen des Algorithmus (Iterationen) erhält man verschiedene Cluster-Lösungen bzw. Arbeitsmarktabgrenzungen mit unterschiedlichem Gütegrad. Abbildung 1 stellt die Werte für die Modularität Q für verschiedene Schwellenwerte und Iterationen für die aggregierte Matrix NEU der Jahre 2006 bis 2008 dar. Die Abszissenwerte zeigen die Anzahl der Arbeitsmarktregionen, die durch das jeweilige Verfahren gebildet werden. Die Linien markieren die höchste Modularität $(\mathrm{Q}=0,8447)$, die als Ergebnis bei der Fusion zu 51 Arbeitsmarktregionen bei einem Schwellenwert von sieben und nach vier Iterationen erreicht wird.

In Abb. 1 werden wichtige Eigenschaften des Verfahrens deutlich: Zum einen werden sehr hohe Modularitätswerte nur erreicht, wenn die Gemeinderegionen zu etwa 30 bis 100 Regionen zusammengefasst werden. Noch gröbere oder deutlich feinere Gliederungen erreichen geringere Werte. Dieses Ergebnis zeigt sich auch, wenn andere Verfahren zur Anwendung kommen (vgl. Kropp 2009). Zum anderen werden die Gemeinderegionen bei niedrigen Schwellenwerten (dunkle Kreise) sehr rasch zu großen Arbeitsmarktregionen zusammengefasst. Dies ist plausibel, weil die Anzahl der Regionen, die mit einem Zentrum mit nur einem geringen Anteil an pendelnden Beschäftigten verbunden sind, größer ist als die Anzahl der Regionen, die stärkere Pendlerverflechtungen

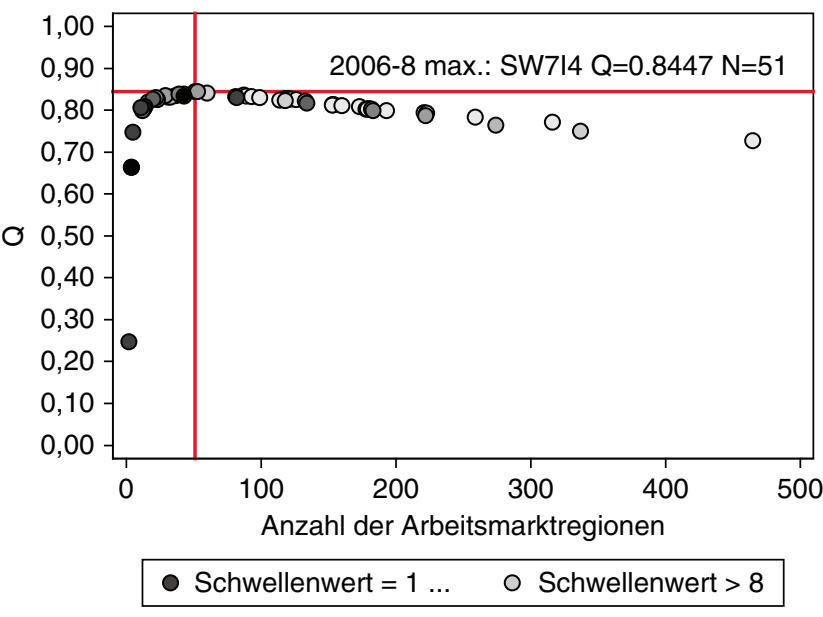

Abb. 1 Modularitätswerte Q bei verschiedenen Schwellenwerten 


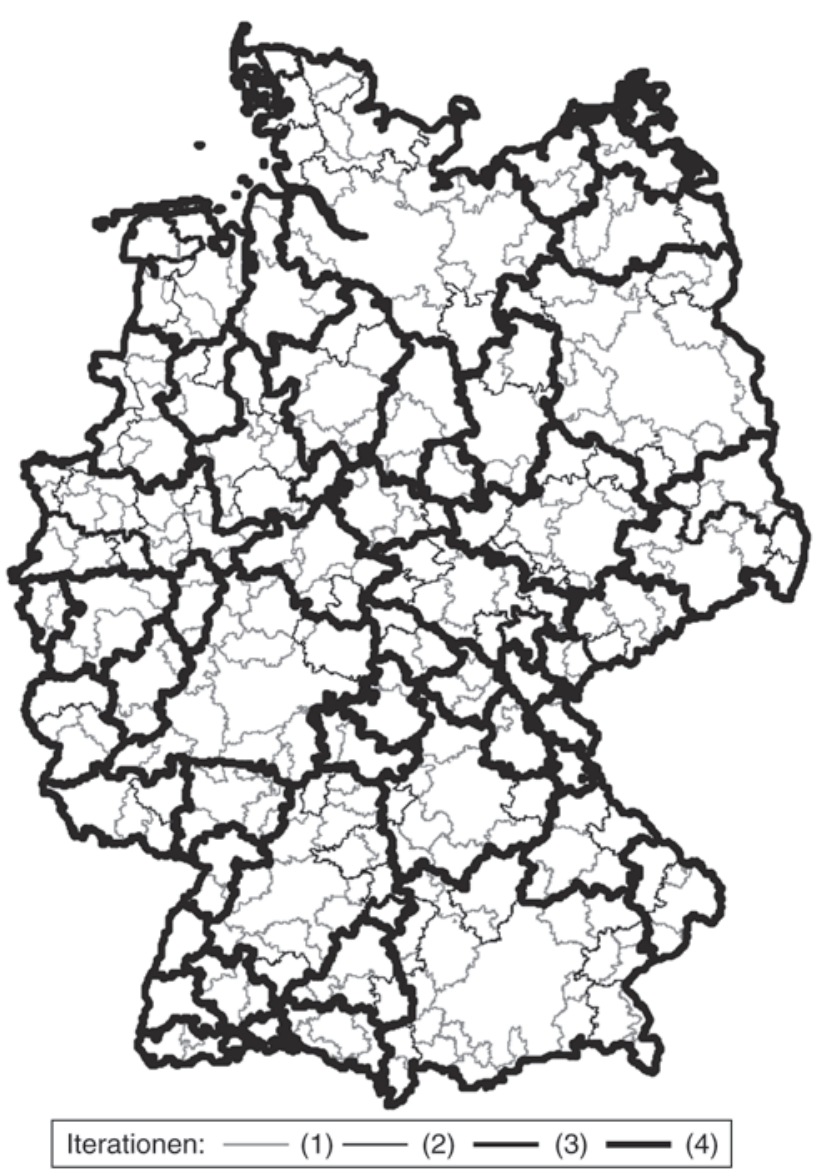

Abb. 2 Bestes analytisches Abgrenzungsergebnis für Gemeinderegionen

mit ihren Zentren aufweisen. Darum finden sich Abgrenzungen mit niedrigen Schwellenwerten eher im linken Bereich der Abbildung. Sehr hohe Schwellenwerte (helle Kreise) führen dagegen dazu, dass der Fusionsprozess stoppt, wenn noch sehr viele Arbeitsmarktregionen vorhanden sind, und noch keine hohen Modularitätswerte erreicht werden.

Die Abgrenzung mit der höchsten Modularität ist in der Karte in Abb. 2 dargestellt (fette Linien). Sie bildet die Basis für die gemeinde- und kreisgenauen Abgrenzungen. Anzumerken ist, dass die Parameter für die Abgrenzung (Schwellenwert und Anzahl der Iterationen) nicht festgelegt, sondern anhand eines Qualitätskriteriums (dem Modularitätswert) ausgewählt wurden. Die ausgewählte Abgrenzung besteht aus $51 \mathrm{sehr}$ größenheterogenen Regionen, die sich möglicherweise weniger für praktische Anwendungen, $\mathrm{z}$. B. in der Regionalpolitik, eignen. Die Abbildung zeigt auch die Ergebnisse der ersten drei Iterationsschritte (dargestellt als dünnere Linien) mit kleineren Arbeitsmarktregionen. So liefert die erste Iteration als Ergebnis den Zusammenschluss zu 337 Arbeitsmarktregionen mit einer Modularität von $\mathrm{Q}=0,7502$. Die zweite Iteration erreicht bereits einen hohen Modularitätswert von $\mathrm{Q}=0,8228$ mit 118 Arbeitsmarktregionen und nach der dritten Iteration unterscheidet sich die
Güte mit $\mathrm{Q}=0,8411$ für 60 Arbeitsmarktregionen nur noch geringfügig von der besten Lösung nach vier Iterationen.

Abbildung 2 zeigt auch, dass monozentrische Regionen wie Hamburg, Berlin oder München besonders schnell wachsen. Dies ist plausibel, weil hier die Pendlerströme häufig auf ein Zentrum hin konzentriert sind. In polyzentrischen Regionen wie dem Ruhrgebiet finden sich klar dominierende Pendlerströme dagegen seltener. Dennoch erscheinen auch hier die Ergebnisse sehr plausibel. Während nach dem ersten Iterationsschritt noch sehr kleinräumige Abgrenzungen dominieren, gibt es bereits nach dem zweiten Iterationsschritt recht großräumige Arbeitsmärkte wie Köln-Bonn, eine Düsseldorfer Region, die auch Mönchengladbach und Krefeld umfasst, sowie eine Ruhr-Region mit Duisburg, Essen und Bochum. Eine vergleichbare Dreiteilung der Rhein-Ruhr-Region schlagen Blotevogel/Schulze (2010: 268) vor. Diese Regionen wachsen im dritten Iterationsschritt weiter. Erst im vierten Iterationsschritt werden die Düsseldorfer und die Ruhr-Region zusammengefasst. Ohne eine adäquate Zusammenfassung sowohl der konzentrierten Pendlerströme in monozentrischen Regionen wie auch der eher ,Netzwerk'-artigen Verflechtungen in polyzentrischen Regionen würden sich keine guten Modularitätswerte für die Abgrenzung erzielen lassen.

Abschließend wurde geprüft, ob jede Gemeinderegion der Arbeitsmarktregion zugeordnet ist, zu der sie die stärkste Pendlerverflechtung hat. Gegebenenfalls wurde die Zuordnung korrigiert. Weil die Änderung für eine Gemeinderegion auch die Zuweisung benachbarter Gemeinderegionen betreffen kann, wurde dieser Korrekturprozess solange wiederholt, bis eine stabile Lösung gefunden war. Insgesamt wurde die Zuweisung von 57 Gemeinderegionen, in denen mit 321.000 Beschäftigten nur ein sehr kleiner Teil aller Beschäftigten tätig ist, geändert. Die Grenzen der Arbeitsmarktregionen verschoben sich nur geringfügig. Die Güte der Abgrenzung erhöhte sich auf $Q=0,8458$.

Eine noch bessere Abgrenzung lie $\beta$ sich mit demselben Optimierungsverfahren auf Gemeindeebene erzielen. Um eine räumlich zusammenhängende Abgrenzung zu erreichen, mussten vier Gemeinden händisch zugeordnet werden. ${ }^{3}$ Außerdem wurde Borkum der Arbeitsmarktregion Oldenburg zugeschlagen. Gegenüber der ursprünglichen Abgrenzung hat sich die Arbeitsmarktzuordnung für 338.000 Beschäftigte geändert. Die Güte dieser Abgrenzung beträgt $\mathrm{Q}=0,8492$. Das ist der beste Wert für eine Abgrenzung in diesem Zeitraum in dieser Studie (vgl. Abb. 3 und Tab. 2).

\footnotetext{
${ }^{3}$ Im Vergleich zu anderen Verfahren ist hervorzuheben, dass die räumliche Kohärenz der Regionen - abgesehen von den vier Gemeinden - ein Ergebnis des Verfahrens ist und nicht durch bestimmte Vorgaben in den Algorithmus implementiert werden musste.
} 
Abb. 3 Vergleich der 50 Arbeitsmarktregionen auf Gemeindeebene mit Kreis- und Bundeslandgrenzen

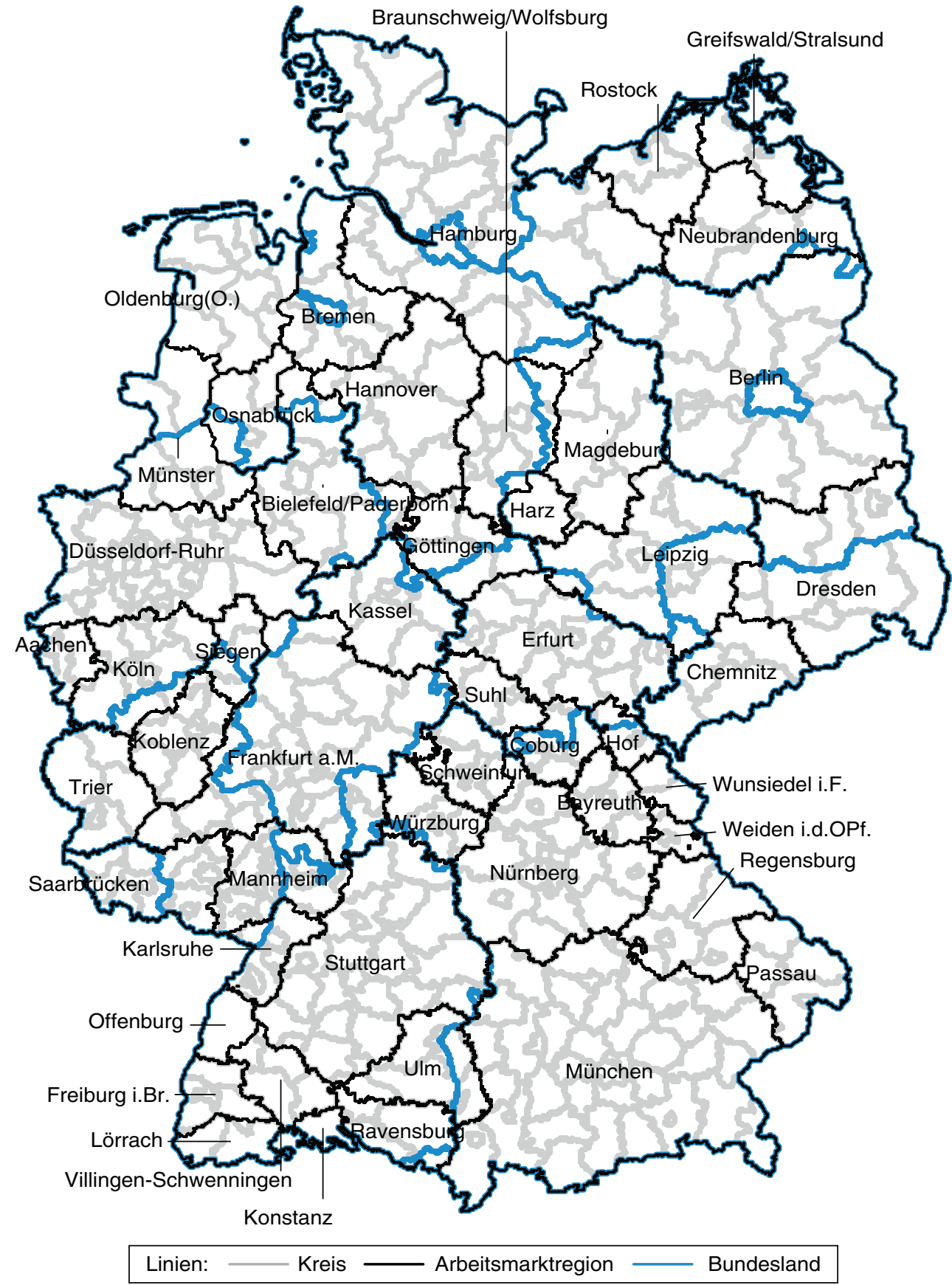

Überraschend sind im Vergleich zu anderen Abgrenzungen die geringe Anzahl von Arbeitsmarktregionen und die Ausmaße der größeren. Arbeitsmarktregionen in der vorliegenden Abgrenzung lassen sich nicht als Arbeitsmarktzentren und ihre unmittelbar angrenzenden Einzugsbereiche charakterisieren, sondern eher als durch direkte und indirekte Pendlerverflechtungen verwobene Großräume. So pendeln zweifellos viele Beschäftigte auch aus dem weiter entfernten Münchner Umland nach München und nehmen dafür weit überdurchschnittliche Entfernungen und Wegezeiten in Kauf (vgl. BBR 2005). Es gibt aber auch zahlreiche Pendler aus dem Umland in benachbarte Arbeitsmarktzen- tren und umgekehrt. Für Regionalanalysen sind die großen Arbeitsmarktregionen möglicherweise nicht differenziert genug. Im Abschn. 5.3 wird darum untersucht, welche lokalen Arbeitsmärkte identifiziert werden können. Die letzten Fusionsschritte geben Hinweise auf solche relativ eigenständige Regionen innerhalb der Arbeitsmarktregionen.

Weil das Verfahren in der vorliegenden Untersuchung auf Pendlerverflechtungen zwischen Gemeinderegionen basiert und die optimierte Abgrenzung gemeindegenau ist, kann die beste analytische Lösung Arbeitsmarktregionen ausweisen, die sich mit Kreis- oder Bundeslandgrenzen überschneiden. Die Abweichungen zwischen diesen gefundenen regio- 
nal verflochtenen Arbeitsmärkten und den administrativen Kreis- bzw. Landesgrenzen zeigt Abb. $3 .{ }^{4}$ Die fetten blauen Linien weisen die Bundeslandgrenzen aus, die dünneren schwarzen Linien die 50 Arbeitsmarktregionen und die hellgrauen Linien zeigen den Zuschnitt der Kreise an.

\subsection{Die zeitliche Stabilität von Arbeitsmarktregionen}

Um die zeitliche Stabilität der gefundenen Abgrenzung von Arbeitsmarktregionen zu prüfen, wurden getrennt für jedes einzelne Jahr zwischen 1993 und 2008 Arbeitsmarktregionen ermittelt. Die höchsten Modularitätswerte der jeweils zehn besten Abgrenzungen sind für jedes Jahr in Abb. 4 dargestellt. Die Werte liegen in allen betrachteten Jahren zwischen 0,84 und 0,88 und damit auf einem sehr hohen Niveau. Auffällig ist die Abnahme der Modularitätswerte im Lauf der Jahre. Dies hängt insbesondere mit dem vermehrten Pendelverhalten - auch über größere Distanzen - sowohl im Tagespendelbereich als auch bei Fernpendlern in den letzten Jahren zusammen (vgl. Behnen/Ott 2006; Einig/Pütz 2007). Weil die Pendlerverflechtungen aus der Summe der Ein- und Auspendler gebildet wurden, spielt die Richtung der Pendlerströme keine Rolle. Die Ergebnisse sollten also relativ robust auch bei Funktionsanreicherungen im Umland sein und relativ unabhängig von Urbanisierungs- oder Reurbanisierungstendenzen, wie sie z. B. Guth/Holz-Rau/ Maciolek et al. (2010) für deutsche Agglomerationsräume beschreiben. Ebenso gibt es Schwankungen im Laufe des betrachteten Zeitraums, denn in einigen Jahren liegen die zehn besten Modularitäten sehr nahe beieinander, in anderen Jahren weisen sie hingegen eine größere Spreizung auf. Ergänzend zu den Ergebnissen für die Jahre 1993 bis 2008 sind diese auch noch für die aggregierten Matrizen der Jahre 1993 bis 1995 (ALT) und für die Jahre 2006 bis 2008 (NEU) sowie für alle Jahre (MW - Mittelwert) ausgewiesen. Auch beim Vergleich der Werte für die Jahreszeiträume NEU und ALT zeigt sich, dass vor über einem Jahrzehnt noch deutlich

\footnotetext{
${ }^{4}$ Die Bezeichnung der Arbeitsmarktregionen (ebenso wie später der lokalen Arbeitsmärkte) richtet sich in der Regel nach der Gemeinde(region) am Endpunkt des Teilgraphen. Nur wenn diese Bezeichnung wenig typisierend für die Arbeitsmarktregion war, weil hier nur wenige Beschäftigte der Arbeitsmarktregionen tätig waren und/oder es gleichwertige Beschäftigungszentren gab, wurden abweichende Bezeichnungen gewählt (z. B. „Braunschweig-Wolfsburg“ statt „Wolfsburg“ oder „Harz" statt „Wernigerode“). Eine Besonderheit bildet die Arbeitsmarktregion „Düsseldorf-Ruhr“. Der Endpunkt des Graphen lag bei Essen, dessen lokaler Arbeitsmarkt zunächst schneller wuchs als der Düsseldorfer (u. a. mit Mülheim und Gladbeck im ersten Iterationsschritt und um die lokalen Arbeitsmärkte Duisburgs und Bochums im zweiten), so dass die Düsseldorfer Region der Essener zugeschlagen wurde. In Düsseldorf sind 11,5 \% aller Beschäftigten der Arbeitsmarktregion tätig und in Essen knapp $7 \%$, so dass man bei beiden Städten schlecht von den dominierenden Zentren der Arbeitsmarktregion sprechen kann.
}

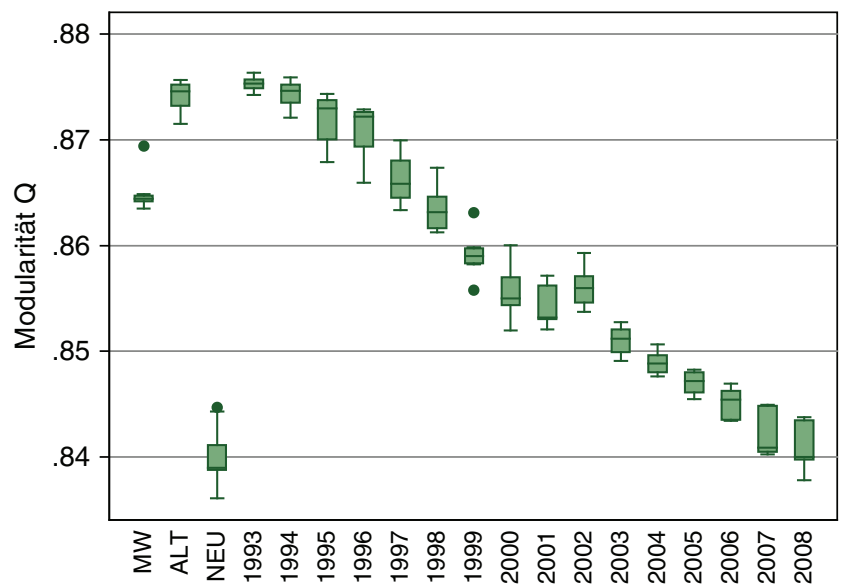

Abb. 4 Die besten zehn Modularitäten pro Jahr

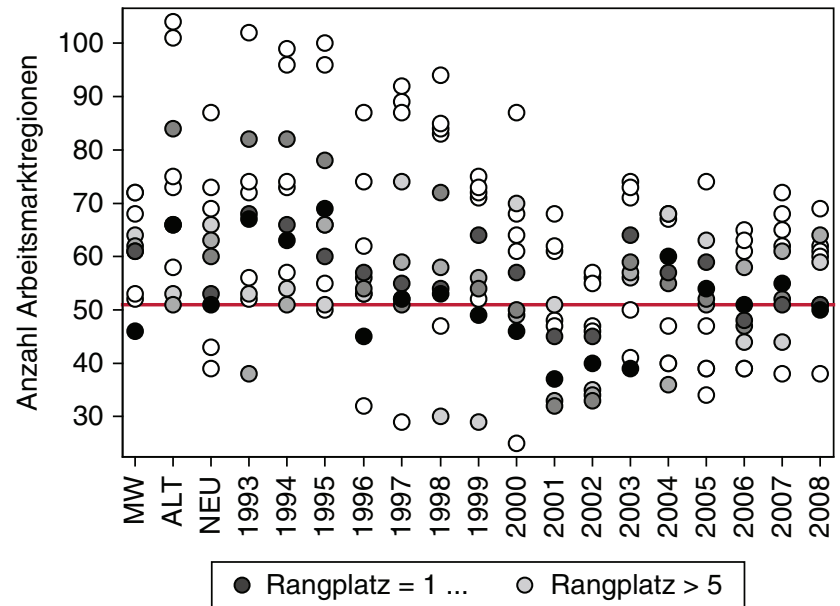

Abb. 5 Zahl der Arbeitsmarktregionen für die zehn besten Modularitäten pro Jahr

höhere Modularitätswerte erreicht wurden als in den Jahren am aktuellen Rand.

Abbildung 5 zeigt für dieselbe Auswahl von Abgrenzungen wie in Abb. 4 die Anzahl der abgegrenzten Regionen. Da sich Pendlerstrukturen in der Regel nur langfristig stark verändern, sollten sich die Abgrenzungen über die Jahre nur wenig unterscheiden. Abbildung 5 gibt Hinweise auf diese zeitliche Stabilität, wobei sich nach dem Jahr 2000 keine Top-10-Abgrenzungen mit über 75 Regionen finden.

Das beste Ergebnis für die Abgrenzung NEU (für die Jahre 2006 bis 2008) auf der Ebene von Gemeinderegionen ist eine Abgrenzung mit 51 Arbeitsmarktregionen. Dass sich in diesem Größenbereich in allen Jahren sehr gute Abgrenzungen finden lassen, deutet darauf hin, dass das Verfahren die zeitliche Stabilität der Pendlerstrukturen adäquat abbilden kann.

Wie gut die zeitliche Stabilität für die beste Lösung mit 51 Arbeitsmarktregionen ausfällt, lässt sich auch als Karte darstellen. Hierfür wurden die 51 Arbeitsmarktregionen für die Jahre 1993-1995 (ALT) und für die Jahre 2006-2008 
Abb. 6 Zeitliche Stabilität der Arbeitsmarktregionen

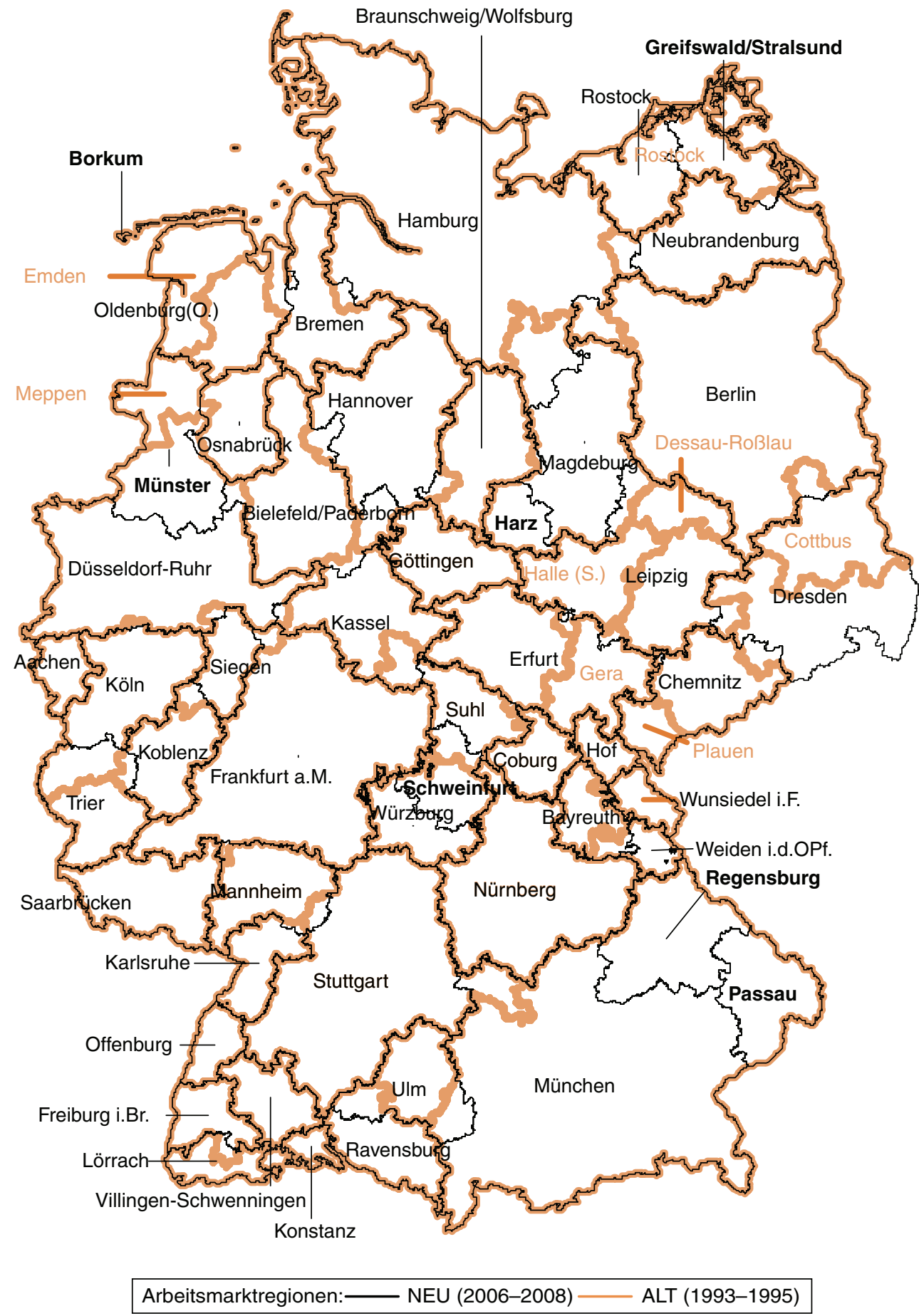

(NEU) in Abb. 6 einander gegenübergestellt. Nur in der Abgrenzung ALT vorkommende Arbeitsmarktregionen sind braun bezeichnet, solche, die nur in NEU vorkommen, schwarz und fett. Bei den meisten Grenzen der Arbeitsmarktregionen lässt sich eine große Übereinstimmung zwischen den beiden Zeiträumen feststellen. Größere Abweichungen gibt es im Südosten der neuen Bundesländer. Für die Jahre 1993 bis 1995 werden hier noch kleinere eigenständige Arbeitsmarktregionen ausgewiesen, wie z. B. Leipzig,
Halle, Dessau-Roßlau. In der Abgrenzung für die Jahre 2006 bis 2008 hingegen gibt es nur noch eine Arbeitsmarktregion Leipzig als beste Lösung, in der diese drei Regionen enthalten sind. Gleiches gilt für Dresden und Cottbus (ALT), zusammengefasst als eine Arbeitsmarktregion Dresden (NEU), Gera und Erfurt (ALT) zu Erfurt (NEU) und Plauen und Chemnitz (ALT) zu Chemnitz (NEU). Kleinräumigere Abgrenzungen existieren für die Jahre 1993 bis 1995 auch im Nordwesten Deutschlands. Oldenburg und Emden wer- 
den für die Jahre 1993 bis 1995 noch als getrennte Arbeitsmarktregionen identifiziert, für die Jahre 2006 bis 2008 jedoch nur noch als eine Region ausgewiesen. Gleiches gilt für Meppen und Münster. Bemerkenswert ist, dass zeitliche Instabilitäten sich in der Regel nicht durch die ,willkürliche ${ }^{6}$ Verschiebung der Grenzen zeigen, sondern darin, dass bestimmte Arbeitsmarktregionen zusammengefasst und andere differenziert werden.

\subsection{Lokale Arbeitsmärkte}

Sowohl der Verlauf des Cluster-Prozesses in Abschn. 5.1 als auch die Analyse der Stabilität der Abgrenzungen im Zeitverlauf geben Hinweise darauf, welche Regionen nur relativ schwach und eher instabil mit dem dominierenden Arbeitsmarktzentrum einer Arbeitsmarktregion verbunden sind. Die wichtigsten dieser lokalen Arbeitsmärkte sollen mit den folgenden Analyseschritten identifiziert werden. Dazu wurden alle „vorläufigen“ Arbeitsmarktregionen aus dem Cluster-Prozess beibehalten, die die Mindestgröße von $50.000^{5}$ Beschäftigten erreichten. Dabei handelt es sich um 70 Regionen. Zusätzlich wurden 50 „Kerne“ der Arbeitsmarktregionen identifiziert, nämlich die Regionen, die bereits im ersten Cluster-Schritt dem späteren Arbeitsmarktzentrum zugeschlagen wurden. Die Grenzen dieser Regionen wurden an die gemeindescharfe Abgrenzung angepasst. Gemeinden, die durch diese Anpassung die Arbeitsmarktregionen gewechselt haben, und alle verbleibenden Gemeinden wurden in einem iterierten Verfahren dem lokalen Arbeitsmarkt oder dem Kern zugewiesen, mit dem sie über ihre Pendlerverflechtungen am stärksten verbunden sind. Das angewandte Verfahren entspricht weitgehend dem auch für die gemeindegenaue Abgrenzung verwendeten. Es wurde allerdings um eine Restriktion mit pro Iteration sinkenden Schwellenwerten ergänzt. Dadurch wird verhindert, dass sehr periphere Gemeinden aufgrund von sehr niedrigen Verflechtungswerten einem Kern oder lokalen Arbeitsmarkt zugewiesen werden. Das Ergebnis ist in Abb. 7 dargestellt. Farblich hervorgehoben sind die lokalen Arbeitsmärkte und (dunkler) die Kernregion. Sie stimmen weitgehend mit den entsprechenden Regionen in Abb. 2 überein. Die weißen Flächen sind die Gemeinden, die dem Kern oder einem lokalen Arbeitsmarkt zuzuweisen waren, weil sie zunächst weder zum lokalen Arbeitsmarkt oder Kern gehören oder nach der Anpassung an die gemeindescharfe Abgrenzung neu zugewiesen werden mussten. Die feinen schwarzen Linien grenzen die lokalen Arbeitsmärkte nach dem beschriebenen Zuweisungsprozess innerhalb ihrer Arbeitsmarktregion ab. So zeigt sich für die Arbeitsmarktregion Hamburg, dass die Kernregion (dunkler eingefärbt) bereits im ersten Cluster-

\footnotetext{
${ }^{5}$ So viele Beschäftigte haben auch die kleinen Arbeitsmarktregionen wie Hof oder Wunsiedel im Fichtelgebirge.
}

Schritt eine sehr große Region umfasst. Daneben erreichen die „vorläufigen“ Arbeitsmarktregionen Flensburg, Kiel, Lübeck und Schwerin die geforderte Mindestgröße von 50.000 Beschäftigten (hellere Färbung innerhalb der feinen schwarzen Abgrenzung). Die restlichen (weißen) Gebiete werden entsprechend ihrer stärksten Anbindung an die dunkler getönten Regionen zugewiesen, z. B. große Teile Nordfrieslands zu Flensburg und von Dithmarschen überwiegend zu Hamburg.

Die lokalen Arbeitsmärkte variieren stark im Hinblick auf ihre Selbstständigkeit. Während der niedrigste Selbstversorgungsgrad mit Beschäftigten für die Arbeitsmarktregionen bei über $75 \%$ liegt, liegen die niedrigsten Werte für die lokalen Arbeitsmärkte bei unter $60 \%$. Vor allem die lokalen Arbeitsmärkte im Ruhrgebiet weisen so niedrige Werte auf, was in Anbetracht der starken Pendlerverflechtungen nicht verwunderlich ist. Dass durch die Bildung der lokalen Arbeitsmärkte wichtige Pendlerströme ignoriert werden, zeigt sich im Sinken der Modularitätswerte auf $Q=0,8026$. Zugleich steigt der Anteil der Pendler an allen Beschäftigten von rund $10,2 \%$ auf $18,0 \%$ (vgl. auch Tab. 2). Die Einordnung der lokalen Arbeitsmärkte in die entsprechenden Arbeitsmarktregionen ist also unumgänglich, um das Ziel der vorliegenden Arbeit, die Pendlerströme durch die Arbeitsmarktabgrenzung möglichst gut ,einzufangen', zu erreichen.

\subsection{Vergleich mit anderen Abgrenzungen anhand deskriptiver Merkmale}

In diesem Abschnitt werden die beschriebenen Arbeitsmarktregionen mit einer kreisscharfen Variante und mit bereits etablierten Abgrenzungen verglichen. Die Zuordnung der Kreise zu Arbeitsmarktregionen bzw. lokalen Arbeitsmärkten kann nach verschiedenen Methoden erfolgen. Für die vorliegende Abgrenzung wurden die Methoden gewählt, die nach den auch zuvor verwendeten Kriterien (Modularität, Pendlerquoten etc.) die besten Ergebnisse lieferten. So wurden die Kreise derjenigen Arbeitsmarktregion zugewiesen, mit der ihre Gemeinden über die stärkste Pendlerverflechtung verbunden waren. ${ }^{6}$ Für knapp $94 \%$ aller Kreise war eine solche Zuordnung ganz klar möglich, weil über zwei Drittel der Pendler der Gemeinden in der Arbeitsmarktregion arbeiten. Nur ein Kreis ist mit weniger als $50 \%$ seiner Gemeindependler mit der zugewiesenen Arbeitsmarktregion verbunden, nämlich die Stadt Worms, deren Pendler zu

\footnotetext{
${ }^{6}$ Die Zuordnung der Kreise zu Arbeitsmarktregionen und lokalen Arbeitsmärkten ist als Excel-Tabelle im Onlinematerial 1 [als Electronic Supplementary Material] publiziert (www.iab.de). Enthalten ist ebenfalls eine Kennziffer zur Güte der Zuordnung (Prozentzahl der Beschäftigten eines Kreises in der Arbeitsmarktregion) und die größte Prozentzahl der Beschäftigten eines Kreises in einer anderen Arbeitsmarktregion.
} 
Abb. 7 Die 50 „Kerne“ der Arbeitsmarktregionen und 70 lokale Arbeitsmärkte (AM)

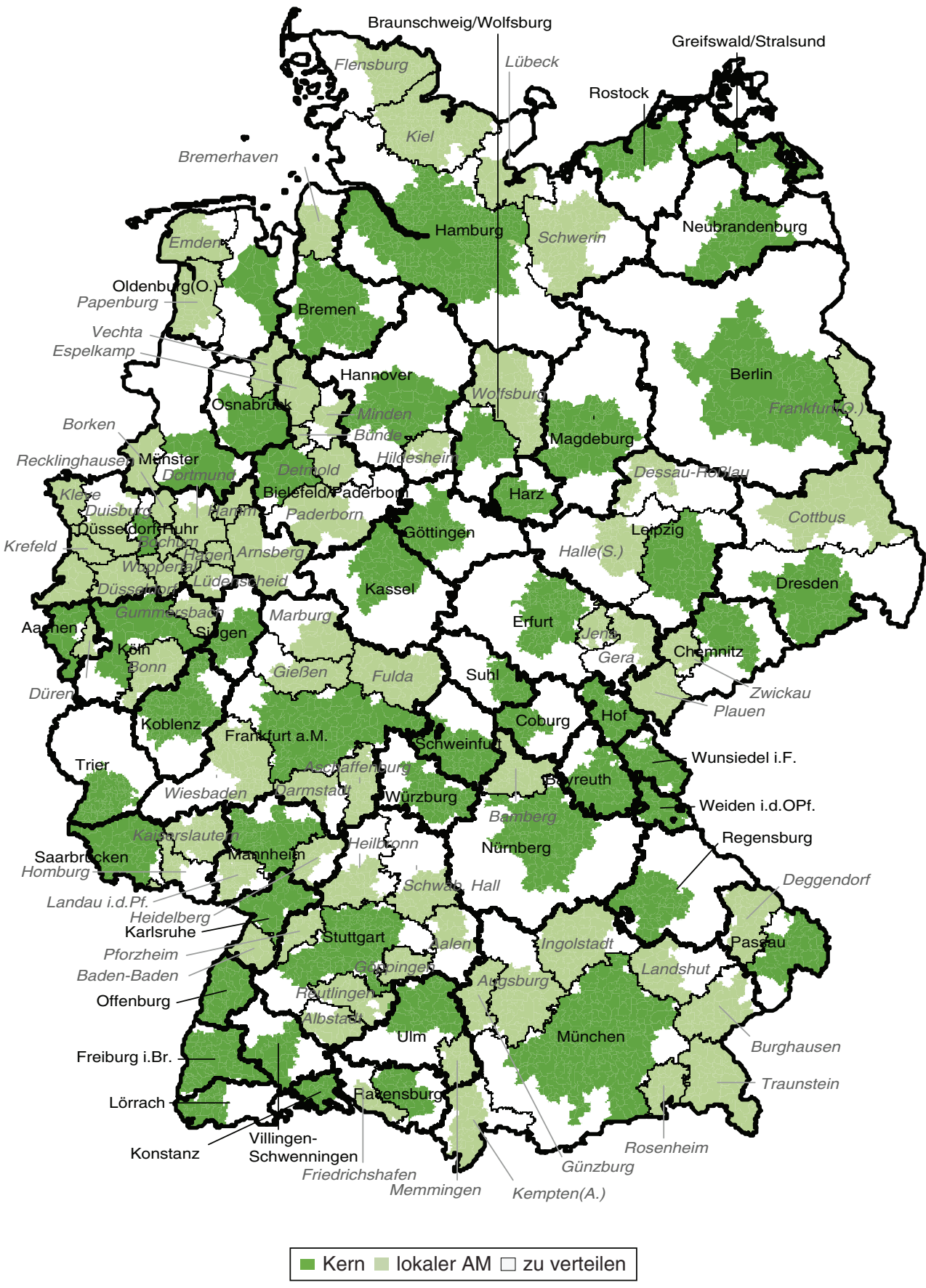

$49 \%$ zur Arbeitsmarktregion Mannheim und zu $41 \%$ zur Arbeitsmarktregion Frankfurt pendeln.

Die Zuordnung der Kreise zu lokalen Arbeitsmärkten erwies als sich deutlich schwieriger. Im Kreis MindenLübbecke liegen die Zentren zweier lokaler Arbeitsmärkte (Espelkamp und Minden), die zusammengelegt werden mussten, so dass im Folgenden nur noch 69 lokale Arbeitsmärkte berücksichtigt werden. Eine wichtige Restriktion bei der Zuordnung der Kreise ist, dass sie in ihrer Arbeitsmarktregion bleiben, auch wenn sie mehr Pendler in andere als in die eigene Arbeitsmarktregion haben. Die Kreise, die das Zentrum eines lokalen Arbeitsmarktes beinhalten, können ebenfalls keinem anderen zugewiesen werden. So ist die Stadt Lübeck mit weniger Pendlern mit dem lokalen Arbeitsmarkt Lübeck verbunden, weil zwischen der Stadt und dem vor allem aus Teilen Ostholsteins bestehendem Umland weniger Pendler unterwegs sind als nach und aus Hamburg. Durch diese Restriktionen sind bereits 119 Kreise als Zentren von Arbeitsmarktregionen bzw. lokalen Arbeitsmärkten gesetzt. Die übrigen wurden dem Kern der Arbeitsmarktregion oder einem lokalen Arbeitsmarkt zugewiesen, mit dem sie die meisten Pendler hatten. Einige lokale 
Tab. 1 Deskriptive Statistiken für die zehn größten und zehn kleinsten Arbeitsmarktregionen. (Quelle: Eigene Berechnung nach Daten des Statistischen Bundesamtes, Volkswirtschaftliche Gesamtrechnung der Länder, und der Bundesagentur für Arbeit)

\begin{tabular}{|c|c|c|c|c|c|c|c|c|}
\hline & $\begin{array}{l}\text { Einwohner } \\
31.12 .2007 \\
\text { in } 1.000\end{array}$ & $\begin{array}{l}\text { BIP } \\
2008 \text { in } \\
\text { Mrd. } €\end{array}$ & $\begin{array}{l}\text { BIP pro Ein- } \\
\text { wohner } 2008 \\
\text { in } 1.000 €\end{array}$ & $\begin{array}{l}\text { Sozialversicherungs- } \\
\text { pflichtig Beschäftigte } \\
\text { am Arbeitsort am } \\
30.06 .2008 \text { in } 1.000\end{array}$ & $\begin{array}{l}\text { Arbeits- } \\
\text { lose im } \\
\text { Juni } 2008 \\
\text { in } 1.000\end{array}$ & $\begin{array}{l}\text { Arbeitslosen- } \\
\text { quote im Juni } \\
2008 \text { in } \%\end{array}$ & $\begin{array}{l}\text { Pendler- } \\
\text { quotient }\end{array}$ & $\begin{array}{l}\text { Selbst- } \\
\text { versor- } \\
\text { gungsgrad }\end{array}$ \\
\hline \multicolumn{9}{|l|}{ Größte Arbeitsmarktregionen: } \\
\hline Düsseldorf-Ruhr & $10.022,0$ & 303,8 & 30,3 & 3115,4 & 464,1 & 9,4 & 100,8 & 91,5 \\
\hline München & $6.166,0$ & 244,9 & 39,7 & 2296,7 & 115,7 & 3,5 & 103,0 & 91,9 \\
\hline Frankfurt am Main & $6.125,1$ & 222,8 & 36,4 & 2197,0 & 189,8 & 6,0 & 105,1 & 88,4 \\
\hline Hamburg & $5.978,5$ & 191,5 & 32,0 & 1972,9 & 235,4 & 7,8 & 101,5 & 93,0 \\
\hline Berlin & $5.457,6$ & 131,9 & 24,2 & 1671,8 & 358,4 & 12,9 & 98,3 & 94,3 \\
\hline Stuttgart & $5.441,5$ & 185,7 & 34,1 & 1966,5 & 107,6 & 3,8 & 101,4 & 92,2 \\
\hline Köln & $3.399,1$ & 109,4 & 32,2 & 1129,0 & 138,4 & 8,2 & 103,2 & 84,2 \\
\hline Bielefeld/Paderborn & $2.412,2$ & 68,4 & 28,4 & 804,8 & 81,8 & 6,8 & 100,7 & 89,7 \\
\hline Nürnberg & $2.348,2$ & 77,9 & 33,2 & 854,1 & 55,6 & 4,4 & 100,9 & 90,5 \\
\hline Leipzig & $2.331,7$ & 51,8 & 22,2 & 730,8 & 172,0 & 14,6 & 94,3 & 91,0 \\
\hline \multicolumn{9}{|l|}{ Kleinste Arbeitsmarktregionen: } \\
\hline Coburg & 365,3 & 10,6 & 29,1 & 131,1 & 10,7 & $\begin{array}{l}5,0 \\
5,5\end{array}$ & 98,4 & 87,0 \\
\hline Greifswald/Stralsund & 349,1 & 7,1 & 20,4 & 107,4 & 25,8 & 14,7 & 93,1 & 91,0 \\
\hline Suhl & 311,0 & 6,3 & 20,3 & 95,9 & 13,6 & 8,3 & 82,8 & 87,1 \\
\hline Bayreuth & 299,5 & 8,7 & 29,1 & 100,8 & 8,0 & 5,1 & 99,0 & 81,8 \\
\hline Konstanz & 272,7 & 8,0 & 29,4 & 82,9 & 5,8 & 4,3 & 98,6 & 87,7 \\
\hline Harz & 226,6 & 4,5 & 19,9 & 64,9 & 15,0 & 13,2 & 84,7 & 87,3 \\
\hline Hof & 185,9 & 5,2 & 27,9 & 63,2 & 6,1 & 6,7 & 104,0 & 77,1 \\
\hline Wunsiedel & 140,2 & 3,6 & 25,4 & 40,9 & 3,7 & 5,4 & 90,3 & 85,0 \\
\hline Weiden & 126,2 & 3,9 & 31,1 & 42,2 & 3,3 & 5,1 & 101,1 & 75,7 \\
\hline
\end{tabular}

Arbeitsmärkte wurden in der kreisgenauen Abgrenzung so beschnitten, dass im verbleibenden Teil deutlich wenigerals 50.000 Beschäftigte tätig waren. Mitunter verloren sie auch einen großen Teil ihres Umlandes, so dass der Selbstversorgungsgrad an Beschäftigten auf bis zu $30 \%$ fiel. In diesen Fällen ist es zweifellos nicht mehr zweckmäßig, von lokalen Arbeitsmärkten zu sprechen. Darum wurden diese lokalen Arbeitsmärkte mit anderen lokalen Arbeitsmärkten oder Arbeitsmarktregionen zusammengelegt. Für das Ruhrgebiet bot sich eine ähnliche Gruppierung an wie von Blotevogel/ Schulze (2010: 268) vorgeschlagen. Dadurch reduzierte sich die Zahl der lokalen Arbeitsmärkte von 69 auf 55.

Die unterschiedlichen Abgrenzungen lassen sich anhand einer Reihe von Merkmalen beschreiben. Eine solche Beschreibung erfolgt in den nächsten Abschnitten beispielhaft für die gemeindegenaue 50-ArbeitsmarktregionenAbgrenzung. Verwendet werden wichtige strukturelle und ökonomische Merkmale, auf die anschließend in aggregierter Form beim Vergleich unterschiedlicher Abgrenzungen zurückgegriffen wird.

Tabelle 1 zeigt eine Reihe von Merkmalen für die zehn größten (einwohnerreichsten) und kleinsten (einwohnerärmsten) Arbeitsmarktregionen der gemeindegenauen Abgrenzungen der Arbeitsmarktregionen. Die größte Region ist die „Düsseldorf-Ruhr"-Region mit 10 Mio. Einwohnern und über 3 Mio. Beschäftigten. Die Wirtschaftsleistung dieser Region beträgt über $12 \%$ der gesamtdeutschen Wirtschaftsleistung. München, Frankfurt am Main und Hamburg bilden mit rund 6 Mio. Einwohnern und einer z. T. nur geringfügig geringeren Wirtschaftskraft die nächst größten Regionen. In Ostdeutschland und Bayern sind einige der kleinsten Arbeitsmarktregionen zu finden. Ein Blick auf die Wirtschaftsleistung pro Einwohner und die Arbeitslosenquoten zeigt, dass die Arbeitsmarktregionen sich auch qualitativ voneinander unterscheiden. So erreichen Greifswald/Stralsund, Suhl und Harz pro Einwohner nur die Hälfte der Wirtschaftsleistung von München. Arbeitslosenquoten von um die $5 \%$ wie in einigen Arbeitsmarktregionen Süddeutschlands (z. B. Weiden, Wunsiedel, Bayreuth und Coburg) sind kaum vergleichbar mit den Arbeitsmarktbedingungen in Ostdeutschland, wo die Arbeitsmarktregion Greifswald/ Stralsund die höchste Quote von 14,7 \% erreicht.

Ein Ziel der angewandten Methodik war es, einen möglichst großen Teil der Pendlerbewegungen in den Arbeitsmarktregionen ,einzufangen ${ }^{6}$. Der Pendlerquotient ${ }^{7}$ der Arbeitsmarktregionen und ihr Selbstversorgungsgrad geben erste Hinweise darauf, wie gut dies gelungen ist. Gerade für die großen Arbeitsmarktregionen zeigen sich sehr ausgeglichene Saldi, so dass man die Regionen weder als ausgesprochene Einpendlerregionen - wie das bei Arbeitsmarktzentren auf Kreisebene häufig der Fall ist - noch als Auspendler-

\footnotetext{
${ }^{7}$ Der Pendlerquotient ist ein Quotient aus Ein- und Auspendlern in Prozent (vgl. Eckey/Kosfeld/Türck 2006: 305, dort als Pendlersaldo bezeichnet). Werte unter 100 kennzeichnen Regionen mit einem Auspendlerüberschuss, Werte über 100 Regionen mit Einpendlerüberschuss.
} 
Tab. 2 Vergleich verschiedener Abgrenzungen hinsichtlich ihrer Güte. (Quelle: Eigene Berechnungen nach Daten der Bundesagentur für Arbeit)

\begin{tabular}{|c|c|c|c|c|c|c|c|c|c|c|c|}
\hline \multirow[t]{2}{*}{ Abgrenzung } & \multirow[t]{2}{*}{$\mathrm{N}$} & \multirow[t]{2}{*}{ Modularität Q } & \multirow{2}{*}{$\frac{\text { Pendler }}{(\%)}$} & \multicolumn{4}{|c|}{ Pendlerquotient } & \multicolumn{4}{|c|}{ Selbstversorgungsgrad } \\
\hline & & & & $\begin{array}{l}\text { Mittel- } \\
\text { wert }\end{array}$ & $\begin{array}{l}\text { Standard- } \\
\text { abweichung }\end{array}$ & $\begin{array}{l}\text { Mini- } \\
\text { mum }\end{array}$ & $\begin{array}{l}\text { Maxi- } \\
\text { mum }\end{array}$ & $\begin{array}{l}\text { Mittel- } \\
\text { wert }\end{array}$ & $\begin{array}{l}\text { Standard- } \\
\text { abweichung }\end{array}$ & $\begin{array}{l}\text { Mini- } \\
\text { mum }\end{array}$ & $\begin{array}{l}\text { Maxi- } \\
\text { mum }\end{array}$ \\
\hline Arbeitsmarktregionen & 50 & 0,8492 & 10,2 & 98,0 & 4,6 & 82,8 & 106,8 & 87,8 & 4,0 & 75,7 & 94,3 \\
\hline $\begin{array}{l}\text { Arbeitsmarktregionen } \\
\text { (Kreise) }\end{array}$ & 50 & 0,8464 & 10,5 & 98,0 & 5,4 & 82,0 & 111,6 & 87,4 & 4,4 & 74,0 & 94,3 \\
\hline $\begin{array}{l}\text { Arbeitsmarktregionen }+ \\
\text { lokale Arbeitsmärkte }\end{array}$ & 120 & 0,8026 & 18,0 & 97,1 & 6,5 & 80,0 & 124,2 & 80,6 & 7,5 & 57,4 & 93,7 \\
\hline $\begin{array}{l}\text { Arbeitsmarktregionen + lokale } \\
\text { Arbeitsmärkte (Kreise) }\end{array}$ & 105 & 0,8150 & 16,3 & 97,1 & 7,1 & 81,9 & 115,6 & 82,2 & 5,9 & 66,8 & 94,3 \\
\hline Regionale Arbeitsmärkte ${ }^{a}$ & 150 & 0,7966 & 18,8 & 95,2 & 8,5 & 58,0 & 122,9 & 80,8 & 5,6 & 58,2 & 90,8 \\
\hline Raumordnungsregionen ${ }^{\mathrm{b}}$ & 96 & 0,7898 & 19,5 & 95,8 & 11,6 & 69,2 & 141,6 & 82,1 & 6,8 & 57,0 & 93,3 \\
\hline Gemeinschaftsaufgabe & 270 & 0,7343 & 25,7 & 92,5 & 13,4 & 58,0 & 136,6 & 75,0 & 7,8 & 47,7 & 89,1 \\
\hline 413 Kreise & 413 & 0,6144 & 38,2 & 98,2 & 35,7 & 40,3 & 301,2 & 63,8 & 15,2 & 22,7 & 90,6 \\
\hline
\end{tabular}

ackey/Kosfeld/Türck (2006)

${ }^{\mathrm{b}} \mathrm{BBSR}(2010)$

regionen charakterisieren kann. Der Selbstversorgungsgrad der Regionen, d. h. das Ausmaß, in dem die Arbeitsplätze in der Region durch in der Region wohnende Personen besetzt werden, ist im Vergleich zu anderen Abgrenzungen ebenfalls hoch (vgl. auch Tab. 2).

In Tab. 2 werden verschiedene Abgrenzungen miteinander verglichen. Hierzu sind neben der Anzahl der abgegrenzten Regionen vor allem Indikatoren wiedergegeben, die die Güte einer Abgrenzung im Hinblick auf die adäquate Erfassung von Pendlerräumen messen. Bereits die Anzahl der abgegrenzten Arbeitsmarktregionen unterscheidet sich deutlich. Die Studie zeigt, dass eine gute Abgrenzung von Pendlerräumen (hohe Modularitätswerte und niedrige Pendlerquoten) etwa 30 bis 75 Arbeitsmarktregionen umfassen sollte (vgl. auch Abb. 5). Die lokalen Arbeitsmärkte und die etablierten Funktionalabgrenzungen haben in der Regel deutlich mehr Regionen; entsprechend ungünstig schneiden diese Abgrenzungen im Hinblick auf die hier verglichenen Indikatoren ab. Dies gilt in besonderem Maße für die Abgrenzung der Gemeinschaftsaufgabe.

Im Vergleich mit den anderen Abgrenzungen zeigt sich die Güte des gewählten Verfahrens. Der Selbstversorgungs$\operatorname{grad}^{8}$ ist für die aktuelle Abgrenzung - auch in der kreisscharfen Variante - deutlich höher als bei den anderen Abgrenzungen. Auch der Streuungswert der Pendlerquotienten (Pendlerquotient: Standardabweichung) ist deutlich geringer als bei anderen Abgrenzungen. Erfreulich ist, dass die kreisscharfe Abgrenzung der gemeindescharfen nur wenig nachsteht, so dass sie in der Regel eine gute Alternative $\mathrm{zu}$ dieser darstellt. Deutlich schlechtere Ergebnisse liefern die Abgrenzungen mit lokalen Arbeitsmärkten. Das ist ein Indiz dafür, dass Arbeitsmarktregionen grundsätzlich als eine Einheit zu behandeln sind, und die Betrachtung

\footnotetext{
${ }^{8}$ Folgende Lage- und Streuungsmaße werden zur Beschreibung der Verteilung ausgewählter Werte verwendet: arithmetischer Mittelwert, Standardabweichung, Minimum und Maximum.
}

von lokalen Arbeitsmärkten im Kontext der zugehörigen Arbeitsmarktregion erfolgen sollte.

Dass hohe Modularitätswerte mit einem niedrigen Pendleranteil, einem ausgeglichenen Pendlerquotienten und einem hohen Selbstversorgungsgrad korrelieren, bestätigt die Eignung des Modularitätsansatzes für die Bewertung von Arbeitsmarktabgrenzungen. ${ }^{9}$ Andere deskriptive Zahlen in Tab. 3 zeigen, wie größenheterogen die abgegrenzten Regionen sind. Arbeitsmarktregionen mit hohen Modularitätswerten erweisen sich als deutlich größenheterogener als die etablierten Funktionalabgrenzungen, für deren Bildung in der Regel auch Mindestgrößen und maximale Pendlerentfernungen zugrunde gelegt wurden. Das belegen die hohen Streuungswerte (Standardabweichung) für die Einwohnerzahl, das Bruttoinlandsprodukt, die Anzahl der sozialversicherungspflichtig Beschäftigten und für die Arbeitslosenzahl. Im Umkehrschluss bedeutet dies auch, dass gute Arbeitsmarktabgrenzungen nicht zugleich größenhomogen sein können, wie es häufig für die praktische Anwendbarkeit gefordert wird.

Abschließend wird gezeigt, dass funktional abgegrenzte Arbeitsmarktregionen auch wirtschaftlich homogenere Räume erzeugen als administrative Abgrenzungen. Quotenwerte wie das Bruttoinlandsprodukt (BIP) pro Einwohner oder die Arbeitslosenquote streuen innerhalb funktionaler Abgrenzungen weniger stark als in administrativ abgegrenzten Regionen. Das zeigen die Streuungswerte für die funktionalen Abgrenzungen und für die Kreise in Tab. 4. Die jeweils erste Spalte gibt nochmals die Streuungswerte zwischen den Regionen wieder, die auch in Tab. 3 zu finden sind. In der jeweils nächsten Spalte sind die Streuungswerte für die entsprechenden Kreisdaten innerhalb der Abgren-

\footnotetext{
${ }^{9}$ Der Vorteil der Verwendung der Modularitätswerte als Maximierungsfunktion gegenüber den anderen Merkmalen liegt vor allem darin, dass gute Abgrenzungen immer eine hohe Modularität haben. Die Maximierung der Binnenpendlerquote dagegen würde eine Fusion aller Regionen nahelegen (vgl. Kropp/Schwengler 2008: 45).
} 
Tab. 3 Vergleich verschiedener Abgrenzungen anhand ausgewählter deskriptiver Statistiken. (Quelle: Eigene Berechnung nach Daten des Statistischen Bundesamtes, Volkswirtschaftliche Gesamtrechnung der Länder, und der Bundesagentur für Arbeit)

\begin{tabular}{|c|c|c|c|c|c|c|c|c|c|c|c|c|}
\hline \multirow[t]{2}{*}{ Abgrenzung } & \multicolumn{4}{|c|}{ Einwohner am 31.12.2007 in 1.000} & \multicolumn{4}{|c|}{ BIP 2008 in Mrd. $€$} & \multicolumn{4}{|c|}{ BIP pro Einwohner 2008 in $1.000 €$} \\
\hline & $\mathrm{Mw}$ & $\mathrm{StAb}$ & Min & Max & $\mathrm{Mw}$ & $\mathrm{StAb}$ & Min & Max & $\mathrm{Mw}$ & $\mathrm{StAb}$ & Min & $\operatorname{Max}$ \\
\hline AMR & $1.648,2$ & $2.039,0$ & 126,2 & $10.022,0$ & 49,9 & 67,4 & 3,6 & 303,8 & 28,4 & 4,8 & 19,7 & 39,7 \\
\hline AMR (Kreise) & $1.648,2$ & $2.024,0$ & 142,8 & $9.845,3$ & 49,9 & 67,1 & 4,0 & 299,3 & 28,4 & 4,8 & 19,7 & 39,6 \\
\hline $\mathrm{AMR}+$ lokale AMR & 686,7 & 774,2 & 112,7 & $5.295,9$ & 20,8 & 26,9 & 3,1 & 153,5 & 28,4 & 5,2 & 19,3 & 46,5 \\
\hline $\begin{array}{l}\text { AMR + lokale AMR } \\
\text { (Kreise) }\end{array}$ & 784,9 & 971,6 & 132,4 & $5.458,4$ & 23,8 & 32,4 & 4,0 & 159,8 & 28,4 & 5,1 & 19,1 & 44,6 \\
\hline $\begin{array}{l}\text { Regionale } \\
\text { Arbeitsmärkte }^{\mathrm{a}}\end{array}$ & 549,4 & 682,0 & 63,6 & $4.443,0$ & 16,6 & 24,2 & 1,4 & 139,9 & 27,7 & 5,8 & 16,6 & 48,3 \\
\hline $\begin{array}{l}\text { Raumordnungs- } \\
\text { regionen }{ }^{\mathrm{b}}\end{array}$ & 858,4 & 623,3 & 227,3 & $3.395,2$ & 26,0 & 24,9 & 4,5 & 129,8 & 28,1 & 6,4 & 18,4 & 50,9 \\
\hline $\begin{array}{l}\text { Gemeinschafts- } \\
\text { aufgabe }\end{array}$ & 305,2 & 399,7 & 63,6 & $3.395,2$ & 9,2 & 15,7 & 1,3 & 126,9 & 26,8 & 6,2 & 15,8 & 52,0 \\
\hline 413 Kreise & 199,5 & 225,1 & 35,2 & $3.395,2$ & 6,0 & 8,7 & 1,0 & 88,6 & 28,6 & 11,1 & 13,2 & 87,7 \\
\hline \multirow[t]{2}{*}{ Abgrenzung } & \multicolumn{4}{|c|}{$\begin{array}{l}\text { Sozialversicherungspflichtig Beschäftigte am } \\
\text { Arbeitsort am } 30.06 .2008 \text { in } 1.000\end{array}$} & \multicolumn{4}{|c|}{ Arbeitslose im Juni 2008 in 1.000} & \multicolumn{4}{|c|}{ Arbeitslosenquote im Juni 2008 in \% } \\
\hline & $\mathrm{Mw}$ & StAb & Min & Max & $\mathrm{Mw}$ & $\mathrm{StAb}$ & Min & $\operatorname{Max}$ & $\mathrm{Mw}$ & $\mathrm{StAb}$ & Min & Max \\
\hline AMR (Gemeinden) & 546,9 & 682,3 & 40,9 & $3.115,4$ & 63,2 & 89,9 & 3,3 & 464,1 & 7,1 & 3,6 & 2,8 & 16,3 \\
\hline AMR (Kreise) & 546,9 & 679,2 & 46,4 & $3.065,9$ & 63,2 & 89,2 & 3,6 & 460,0 & 7,1 & 3,6 & 2,8 & 16,3 \\
\hline AMR + lokale AMR & 227,9 & 272,3 & 32,4 & $1.621,1$ & 26,3 & 38,8 & 1,5 & 346,0 & 7,0 & 3,5 & 2,2 & 16,3 \\
\hline $\begin{array}{l}\text { AMR+ lokale AMR } \\
\text { (Kreise) }\end{array}$ & 260,4 & 329,0 & 46,4 & $1.671,6$ & 30,1 & 49,3 & 2,0 & 358,5 & 6,8 & 3,4 & 2,2 & 16,3 \\
\hline $\begin{array}{l}\text { Regionale } \\
\text { Arbeitsmärkte }^{\mathrm{a}}\end{array}$ & 182,3 & 242,1 & 15,2 & $1.349,0$ & 21,1 & 32,8 & 1,4 & 293,0 & 7,4 & 4,1 & 2,1 & 18,6 \\
\hline $\begin{array}{l}\text { Raumordnungs- } \\
\text { regionen }^{\mathrm{b}}\end{array}$ & 284,8 & 239,6 & 61,2 & $1.131,0$ & 32,9 & 31,9 & 5,6 & 229,0 & 7,5 & 3,7 & 2,3 & 15,7 \\
\hline $\begin{array}{l}\text { Gemeinschafts- } \\
\text { aufgabe }\end{array}$ & 101,3 & 153,6 & 15,2 & $1.100,3$ & 11,7 & 19,1 & 1,0 & 229,0 & 7,3 & 3,9 & 2,1 & 18,9 \\
\hline 413 Kreise & 66,2 & 88,7 & 11,8 & $1.080,6$ & 7,6 & 13,4 & 0,7 & 229,0 & 7,2 & 3,9 & 1,4 & 20,0 \\
\hline
\end{tabular}

Mw Mittelwert, StAb Standardabweichung, Min Minimum, Max Maximum, AMR Arbeitsmarktregionen

${ }^{a}$ Eckey/Kosfeld/Türck (2006)

bBBSR (2010)

Tab. 4 Streuungswerte (Standardabweichungen) zwischen abgegrenzten Regionen und für Kreise innerhalb der Abgrenzungen. (Quelle: Eigene Berechnungen nach Daten des Statistischen Bundesamtes, Volkswirtschaftliche Gesamtrechnung der Länder, und der Bundesagentur für Arbeit)

\begin{tabular}{|c|c|c|c|c|}
\hline & \multicolumn{2}{|c|}{$\begin{array}{l}\text { BIP pro Einwohner 2008, } \\
\text { Standardabweichung }\end{array}$} & \multicolumn{2}{|c|}{$\begin{array}{l}\text { Arbeitslosenquote im Juni } 2008 \text { in \%, } \\
\text { Standardabweichung }\end{array}$} \\
\hline & $\begin{array}{l}\text { Zwischen } \\
\text { Regionen }\end{array}$ & $\begin{array}{l}\text { zwischen Kreisen in } \\
\text { den Regionen }\end{array}$ & $\begin{array}{l}\text { Zwischen } \\
\text { Regionen }\end{array}$ & $\begin{array}{l}\text { zwischen Kreisen in } \\
\text { den Regionen }\end{array}$ \\
\hline Arbeitsmarktregionen (Kreise) & 4,8 & 9,1 & 3,6 & 1,8 \\
\hline Arbeitsmarktregionen + lokale Arbeitsmärkte (Kreise) & 5,1 & 9,4 & 3,4 & 1,8 \\
\hline Regionale Arbeitsmärkte & 5,8 & 9,1 & 4,1 & 1,8 \\
\hline Raumordnungsregionen ${ }^{\mathrm{b}}$ & 6,4 & 7,8 & 3,7 & 1,7 \\
\hline Abgrenzung Gemeinschaftsaufgabe & 6,2 & 10,7 & 3,9 & 2,0 \\
\hline 413 Kreise & 11,1 & 1 & 3,9 & 1 \\
\hline
\end{tabular}

${ }^{\mathrm{a}}$ Eckey/Kosfeld/Türck (2006)

${ }^{\mathrm{b} B B S R}(2010)$

zung enthalten. Weil die Berechnungen auf Kreisdaten beruhen, sind hier nur kreisgenaue Abgrenzungen berücksichtigt. Die Werte für die neu entwickelten Abgrenzungen liefern dabei nicht immer die besten Werte, aber die niedrigen Streuungswerte sind durchaus bemerkenswert, weil die relativ homogenen Regionen mit einer sehr groben $-\mathrm{d}$. h. vergleichsweise wenige Regionen umfassenden - Abgrenzung erzeugt werden konnten.

\section{Fazit}

Die Bedeutung räumlicher ökonomischer Prozesse für die Wirtschaftsentwicklung wurde in der ökonomischen Theorie schon früh betont und ist in den letzten Jahren im Zusammenhang mit der Neuen Ökonomischen Geographie wieder stärker diskutiert worden. Das Konzept funktionaler Regio- 
nen versucht die Realität räumlicher ökonomischer Prozesse so gut wie möglich abzubilden, indem sie Regionen so abgrenzt, dass sich der Großteil der ökonomischen Aktivitäten innerhalb ihrer Grenzen konzentriert (Smart 1974: 261; Coombes/Green/Openshaw 1986: 944; van der Laan/ Schalke 2001: 205; Bongaerts/Cörvers/Hensen 2004: 2).

Die vorliegende Arbeit nimmt auf Basis der graphentheoretischen Methode Abgrenzungen von Arbeitsmarktregionen vor. Datengrundlage hierfür waren die Wohn- und Arbeitsorte aller sozialversicherungspflichtig Beschäftigten auf Gemeindeebene in Deutschland für die Jahre 1993 bis 2008. Das von Rabino und Occelli (1997) entwickelte Verfahren verband den graphentheoretischen Ansatz mit dem in der Regionalforschung zur Abgrenzung von Regionen gut etablierten Schwellenwertverfahren. Um es großflächig für ganz Deutschland zu nutzen, wird das Agglomerationsverfahren iteriert angewandt, wobei die Pendlerverflechtungen zwischen den aggregierten Teilgraphen neu berechnet werden. Das graphentheoretische Verfahren wird somit in ein hierarchisches Cluster-Verfahren integriert. Diese Methodik hat sich in einem Methodenvergleich gut bewährt (vgl. Kropp/Schwengler 2008) und konnte inzwischen weiter verbessert werden. Insbesondere erlaubt ein abschließender Optimierungsalgorithmus Korrekturen für suboptimale Zuordnungen von Regionen zu Arbeitsmarktregionen, wie sie sich für hierarchische Verfahren nicht gänzlich ausschließen lassen, sowie die Anpassung an unterschiedliche Gebietsstände.

Zur Bewertung der Güte der gefundenen Abgrenzungen wurde die Modularität Q aus der Netzwerkforschung herangezogen. Dieses Maß vergleicht die Netzwerkstruktur der Pendlerverflechtungen mit einem geeigneten Nullmodell und erlaubt Schlussfolgerungen, wie stark eine vorgefundene Struktur vom Nullmodell abweicht. Der Vergleich mit anderen Gütemaßen für funktionale Abgrenzungen, wie dem Selbstversorgungsgrad oder den Pendlerquoten, zeigt die Validität der Bewertung durch das Modularitätsmaß.

Im Zeitraum von 1993 bis 2008 wurde für jedes einzelne Jahr die beste Abgrenzung mit einer vergleichbaren Anzahl an Arbeitsmarktregionen zur aktuellen Abgrenzung gesucht und die so gefundenen Ergebnisse miteinander verglichen. Dabei zeigte sich eine hohe zeitliche Stabilität zwischen den Lösungen, auch wenn die Güte für die ersten Jahre höher lag als am aktuellen Rand. Dies mag damit zusammenhängen, dass die Pendelzeiten und Pendelentfernungen im Laufe der Jahre deutlich zugenommen haben und sich Arbeitsmarktregionen darum nicht mehr so klar voneinander ,trennen' lassen. In dieser Untersuchung wurden für den Jahreszeitraum von 1993 bis 1995 insbesondere im Südosten der neuen Bundesländer sowie im Nordwesten der alten Bundesländer kleinräumigere Arbeitsmarktregionen als optimale Lösung ausgewiesen. Für den Zeitraum der Jahre 2006 bis 2008 lieferte das Verfahren hingegen als optimale Abgrenzung in diesen Regionen größere lokale Arbeitsmärkte, was die Vermutung für längere Pendeldistanzen bestätigen würde. Generell lässt sich eine hohe Stabilität für die Metropolen der Republik zeigen. Schwieriger ist eine stabile Zuordnung in Regionen ohne ein klar dominierendes Zentrum oder im Randbereich der großen Arbeitsmarktregionen.

Für die Jahre 2006 bis 2008 konnte eine optimale Abgrenzung mit 50 Arbeitsmarktregionen identifiziert werden. Diese Abgrenzung liegt sowohl auf Gemeinde- wie auf Kreisebene vor. 50 Arbeitsmarktregionen sind deutlich weniger als bei den bisher entwickelten und verwendeten Abgrenzungen, wie z. B. der aktuellen Abgrenzung von Eckey/Kosfeld/ Türck (2006) mit 150 Arbeitsmarktregionen, der derzeit verwendeten 270 Arbeitsmarktregionen der Gemeinschaftsaufgabe „Verbesserung der regionalen Wirtschaftsstruktur“ oder der 96 Raumordnungsregionen des Bundesinstituts für Bau-, Stadt- und Raumforschung (BBSR). Zudem weist die hier präsentierte analytisch beste Lösung sehr größenheterogene Regionen auf. Dabei stellt sich insbesondere für kleine Arbeitsmarktregionen die Frage, ob diese nicht zu klein sind, um wirtschaftlich prosperieren zu können und ob sie z. B. durch Infrastrukturmaßnahmen besser an größere Arbeitsmarktregionen angebunden werden sollten.

Insbesondere die großen Arbeitsmarktregionen lassen sich zweifellos nicht als die unmittelbare Pendlerregion des dominierenden Zentrums charakterisieren. Hier spielen auch indirekte Pendlerverflechtungen eine wichtige Rolle. So ist z. B. das Münchner Umland sehr weiträumig mit der Stadt München verbunden, aber auch weiter entfernte Regionen, die sich teilweise selbst als lokale Arbeitsmärkte charakterisieren lassen, sind wiederum mit dem Umland verflochten. Dennoch haben auch die großen Arbeitsmarktregionen einen gemeinsamen Arbeitsmarkt. Durch die (indirekten) Verflechtungen werden auch entferntere Regionen derselben Arbeitsmarktregionen mittelfristig mehr von starken Veränderungen in einer Region der Arbeitsmarktregionen betroffen sein als Regionen außerhalb der Arbeitsmarktregionen.

Um den Erfordernissen differenzierterer Regionalanalysen zu entsprechen, wurden insbesondere für die großen Arbeitsmarktregionen relativ eigenständige lokale Arbeitsmärkte identifiziert, die neben den 50 Kernregionen der Arbeitsmarktregionen bestehen. Die Güte einer solchen Abgrenzung ist jedoch deutlich geringer als die ursprüngliche 50-Regionen-Abgrenzung. Darum sollte die Entwicklung auf lokalen Arbeitsmärkten stets im Kontext der Arbeitsmarktregion betrachtet werden, zu der sie gehören. Dagegen weist die kreisscharfe Variante der Abgrenzung ähnlich gute Gütemaße auf wie die gemeindescharfe. Nur für einige Kreise zeigten sich Zuweisungsprobleme. Ähnlich unproblematisch dürfte es sein, sehr kleine Arbeitsmarktregionen mit benachbarten größeren zu fusionieren.

Beim Vergleich der Abgrenzungsvarianten (mit und ohne lokale Arbeitsmärkte, gemeinde- oder kreisscharf) 
und beim Vergleich mit bestehenden Abgrenzungen zeigte sich die Nutzbarkeit der verwendeten Gütemaße. Abgrenzungen mit hohen (d. h. günstigen) Modularitätswerten hatten weniger Pendler aus den Arbeitsmarktregionen heraus, ausgeglichenere Pendlerquoten und einen hohen Selbstversorgungsgrad mit Beschäftigten. Funktional abgegrenzte Arbeitsmarktregionen bilden wirtschaftlich homogenere Räume als administrative Abgrenzungen. Dies konnte für das Bruttoinlandsprodukt pro Einwohner und die Arbeitslosenquote gezeigt werden. Diese Indikatoren streuen innerhalb funktionaler Abgrenzungen weniger stark als zwischen Kreisen. Die Werte für die neu entwickelten Abgrenzungen liefern dabei nicht immer die besten Werte, aber die niedrigen Streuungswerte sind durchaus bemerkenswert, weil die relativ homogenen Regionen mit einer sehr groben $-\mathrm{d}$. h. vergleichsweise wenige Regionen umfassenden - Abgrenzung erzeugt werden konnten.

Dass das Bruttoinlandsprodukt pro Einwohner innerhalb von Arbeitsmarktregionen weniger streut als zwischen administrativen Regionen, kann ein Hinweis darauf sein, dass Grenzen von Arbeitsmarktregionen nicht nur für Arbeitsmarktprozesse, sondern auch für andere wirtschaftliche Interaktionen, wie z. B. lokale Gütermärkte oder Wohnungsmärkte, relevant sind.

Gute Modularitätswerte erzielten Abgrenzungen mit wenigen Regionen. Eine optimale Abgrenzung der Pendlerströme erfordert offensichtlich etwa 30 bis 75 Regionen. Differenziertere Abgrenzungen durchschneiden zwangsläufig wichtige Pendlerströme. Dies zeigt sich auch in deutlich höheren Pendlerquoten bei Abgrenzungen mit vielen Regionen, insbesondere bei der Abgrenzung für die Gemeinschaftsaufgabe. Die Ergebnisse der vorliegenden Studie zeigen auch die Diskrepanz zwischen administrativen und funktionalen Abgrenzungen. Dies gilt in besonderem Maße für die Stadtstaaten. Deren Arbeitsmarkt ist in jedem Fall weit größer als die eigenen Bundeslandgrenzen. Ohne eine koordinierte, bundeslandübergreifende Arbeitsmarkt- und Wirtschaftspolitik, wie sie z. B. in den entsprechenden Metropolregionen institutionalisiert ist, lassen sich wichtige Arbeitsmarktthemen nicht erfolgreich angehen. Unterstützt zum Beispiel ein Stadtstaat Beschäftigungsaufbau mit Fördergeldern, so sinkt dadurch möglicherweise nicht die Arbeitslosigkeit im Stadtstaat, sondern im Umland. Ebenso können Qualifizierungsmaßnahmen in einem Stadtstaat zu Verdrängungsprozessen im Umland und zu einer erhöhten Arbeitslosigkeit dort führen. Dagegen lassen sich durch die ökonomische Konzentration von Entwicklungspotenzialen in Regionen und ihren angrenzenden Einzugsbereichen Agglomerationsvorteile so generieren, dass sie die Wettbewerbsfähigkeit der gesamten Arbeitsmarktregion stärken.

Häufig folgen die Grenzen der Arbeitsmarktregionen recht deutlich den Kreis- oder Bundeslandgrenzen. Das macht die Umrechnung der gemeindescharfen Abgrenzung auf Kreisebene möglich. Für Kreise, die durch Arbeitsmarktgrenzen durchschnitten werden, gilt es jedoch zu prüfen, ob unterschiedliche Politikansätze in den entsprechenden Kreisteilen nötig sind.

Die vorliegende Studie hat unterschiedliche Arbeitsmarktregionen für Deutschland abgegrenzt. Daraus ergeben sich weitere Fragen: Wie stark unterscheiden sich die Arbeitsmarktregionen voneinander? Erfordern sie den Einsatz unterschiedlicher Instrumente in der Arbeitsmarktund Wirtschaftspolitik? Welche Voraussetzung bringen die Arbeitsmarktregionen mit für wirtschaftliche Entwicklung? Inwiefern sind sie voneinander abhängig bzw. selbstständig? Wie kann die Zusammenarbeit von unterschiedlichen Administrationen gefördert werden? Die Autoren hoffen, mit ihrer Abgrenzung eine gute Basis für die Beantwortung solcher Fragen gelegt zu haben.

\section{Literatur}

ARL (Akademie für Raumforschung und Landesplanung) (2007): Metropolregionen - Innovation, Wettbewerb, Handlungsfähigkeit. Hannover. $=$ Positionspapier aus der ARL, Nr. 71.

BBSR (Bundesinstitut für Bau-, Stadt- und Raumforschung) (2009): Positionierung Europäischer Metropolregionen in Deutschland. Bonn. = BBSR-Berichte KOMPAKT 3/2009.

BBSR (Bundesinstitut für Bau-, Stadt- und Raumforschung) (2010): Raumordnungsregionen (Analyseräume). Online unter: http:// www.bbsr.bund.de/cln_016/nn_103086/BBSR/DE/Raumbeobachtung/Werkzeuge/Raumabgrenzungen/Raumordnungsregionen/raumordnungsregionen.html; letzter Zugriff am 01.04.2010.

BBR (Bundesamt für Bauwesen und Raumordnung) (2005): Raumordnungsbericht 2005. Bonn.

Behnen, T.; Ott, E. (2006): Arbeitskräftemobilität - Fernpendler und ihre Lebenssituation. In: Leibniz-Institut für Länderkunde (Hrsg.): Nationalatlas Bundesrepublik Deutschland, Band 7: Arbeit und Lebensstandard. München, 56-59.

Binder, J.; Schwengler, B. (2006): Arbeitsmarktregionen im Raum Berlin und Brandenburg - Kritische Überprüfung der bisher gültigen Arbeitsmarktregionen und Vorschläge für einen Neuzuschnitt. Nürnberg. = IAB-Forschungsbericht Nr. 4/2006.

Blotevogel, H. H. (1996): Zur Kontroverse um den Stellenwert des Zentrale-Orte-Konzepts in der Raumordnungspolitik heute. In: Informationen zur Raumentwicklung 10, 647-657.

Blotevogel, H. H. (2005): Zentrale Orte. In: Akademie für Raumforschung und Landesplanung (ARL) (Hrsg.): Handwörterbuch der Raumordnung. Hannover, 1307-1315.

Blotevogel, H. H.; Schulze, K. (2010): 1 oder 2 oder 3? Zur Konstituierung möglicher Metropolregionen an Rhein und Ruhr. In: Raumforschung und Raumordnung 68, 4, 255-270.

Bode, E. (2008): Delineating Metropolitan Areas using Land Prices. In: Journal of Regional Science 48, 1, 131-163.

Bongaerts, D.; Cörvers, F.; Hensen, M. (2004): The Delimitation and Coherence of Functional and Administrative Regions. The Hague. $=$ Ministry of Economic Affairs, Research Series 04019.

Brandes, U.; Delling, D.; Gaertler, M.; Görke, R.; Hoefer, M.; Nikoloski, Z.; Wagner, D. (2008): On Modularity Clustering. In: IEEE Transactions on Knowledge and Data Engineering 20, 2, 172-188. 
Budde, R.; Eckey, H.-F.; Klemmer, P. (1993): Vorschlag für die Abgrenzung von Arbeitsmarktregionen in den neuen Bundesländern. Gutachten des Rheinisch-Westfälischen Instituts für Wirtschaftsforschung. Essen.

Budde, R.; Eckey, H.-F.; Schrumpf, H. (1995): Vorschlag für die Abgrenzung von Arbeitsmärkten in den neuen Bundesländern auf der Grundlage der durchgeführten Regionalreformen. Gutachten des Rheinisch-Westfälischen Instituts für Wirtschaftsforschung. Essen.

Bundesagentur für Arbeit (2008): Der Arbeits- und Ausbildungsmarkt in Deutschland. Monatsbericht Dezember und das Jahr 2008. Nürnberg.

Casado-Díaz, J. M. (2000): Local labour market areas in Spain: a case study. In: Regional Studies 34, 9, 843-856.

Christaller, W. (1933): Die zentralen Orte in Süddeutschland. Jena.

Cörvers, F.; Hensen, M.; Bongaerts, D. (2009): Delimitation and Coherence of Functional and Administrative Regions. In: Regional Studies 43, 1, 19-31.

Coombes, M. G.; Green, A. E.; Openshaw, S. (1986): An efficient algorithm to generate official statistical reporting areas: the case of the 1984 travel-to-work areas revision in Britain. In: Journal of the Operational Research Society 37, 10, 943-953.

Dauth, W. (2010): Agglomeration and regional employment growth. Nürnberg. = IAB-Discussion Paper 07/2010.

Eckey, H.-F. (1988): Abgrenzung regionaler Arbeitsmärkte. In: Raumforschung und Raumordnung 46, 1-2, 24-33.

Eckey, H.-F. (2001): Der wirtschaftliche Entwicklungsstand in den Regionen des vereinigten Deutschlands. Kassel. = Volkswirtschaftliche Diskussionsbeiträge der Universität Kassel, Nr. 20/01.

Eckey H.-F.; Klemmer, P. (1991): Neuabgrenzung von Arbeitsmarktregionen für die Zwecke der regionalen Wirtschaftspolitik. In: Informationen zur Raumentwicklung 9/10, 569-577.

Eckey, H.-F.; Kosfeld, R.; Türck, M. (2006): Abgrenzung deutscher Arbeitsmarktregionen. In: Raumforschung und Raumordnung 64, 4, 299-309.

Einig, K.; Pütz, T. (2007): Regionale Dynamik der Pendlergesellschaft. Entwicklung von Verflechtungsmustern und Pendeldistanzen. In: Informationen zur Raumentwicklung 2/3, 73-91.

Fortunato, S. (2010): Community detection in graphs. In: Physics Reports 486, 3-5, 75-174.

Gorman, S. P.; Patuelli, R.; Reggiani, A.; Nijkamp, P.; Kulkarni, R.; Haag, G. (2007): An Application of Complex Network Theory to German Commuting Patterns. In: Friesz, T. L (Hrsg.): Network Science, Nonlinear Science and Infrastructure Systems. Berlin, Heidelberg, New York, 167-185.

Granato, N.; Farhauer, O. (2007): Die Abgrenzung von Arbeitsmarktregionen: Gütekriterien und -maßzahlen. Berlin. $=$ Wirtschaftswissenschaftliche Dokumentation, Diskussionspapier 2007/02, Technische Universität Berlin.

Guth, D.; Holz-Rau, C.; Maciolek, M.; Scheiner, J. (2010): Beschäftigungssuburbanisierung, Siedlungsstruktur und Berufspendelverkehr: Ergebnisse für deutsche Agglomerationsräume 1999-2007. In: Raumforschung und Raumordnung 68, 4, 283-295.

Haag, G.; Binder, J. (2001): Endbericht zum Forschungsprojekt „Vergleichende Untersuchung der Wirkungszusammenhänge zwischen Pendelverkehr und der räumlichen Organisationsstruktur der Region Stuttgart und der Provinz Turin“. Sonderauswertung der Ergebnisse des VIGONI-Projekts im Auftrag des Verbandes Region Stuttgart. Stuttgart.
Hensen, M.; Cörvers, F. (2003): The regionalization of labour markets by modelling commuting behaviour. Online unter: http://www. roa.unimaas.nl/seminars/pdf2005/M.Hensen.pdf; letzter Zugriff am 26.01.2010.

Karlsson, C.; Olsson, M. (2006): The identification of functional regions: theory, methods, and applications. In: Annals of Regional Science 40, 1, 1-18.

Klemmer, P.; Kraemer, D. (1975): Regionale Arbeitsmärkte. Ein Abgrenzungsvorschlag für die Bundesrepublik Deutschland. Bochum. = Beiträge zur Struktur- und Konjunkturforschung, Band 1.

Kosfeld, R.; Eckey, H.-F.; Türck, M. (2008): New economic geography and regional price level. In: Jahrbuch für Regionalwissenschaft $28,1,43-60$.

Kropp, P. (2009): Die Abgrenzung der Arbeitsmarktregion Bremen. Nürnberg. = IAB-Regional 3/2009.

Kropp, P.; Schwengler, B. (2008): Abgrenzung von Wirtschaftsräumen auf der Grundlage von Pendlerverflechtungen. Ein Methodenvergleich. Nürnberg. = IAB-Discussion Paper 41/2008.

Krugman, P. (1991): Geography and trade. Cambridge (Mass.).

Lösch, A. (1940): Die räumliche Ordnung der Wirtschaft. Jena.

Madelin, M.; Grasland, C.; Mathian, H.; Sanders, L.; Vincent, J.-M. (2009): Das „MAUP“: Modifiable Areal Unit - Problem oder Fortschritt? In: Informationen zur Raumentwicklung 10/11, 645-660.

Newman, M. E. J.; Girvan, M. (2004): Finding and evaluating community structure in networks. In: Physical Review E 69, 2, 026113.

Nystuen, J. D.; Dacey, M. F. (1961): A Graph Theory Interpretation of Nodal Regions. In: Papers and Proceedings of the Regional Science Association 7, 1, 29-42.

ONS (Office for National Statistics); Coombes, M. G. (1998): 1991based Travel-to-Work Areas. London.

Openshaw, S. (1984): The modifiable areal unit problem. Norwich. $=$ Concepts and Techniques in Modern Geography, No. 38.

Rabino, G. A.; Occelli, S. (1997): Understanding spatial structure from network data: theoretical considerations and applications. In: Cybergeo. European Journal of Geography. Online unter: http://www.cybergeo.eu/index2199.html; letzter Zugriff am 27.01.2010.

Smart, M. W. (1974): Labour market areas: Uses and definition. In: Progress in Planning 32, 2, 239-353.

Sternberg, R. (2001): „New Economic Geography“ und „Neue regionale Wachstumstheorie“" aus wirtschaftsgeographischer Sicht. In: Zeitschrift für Wirtschaftsgeographie 45, 3/4, 159-180.

Tolbert, C. M.; Killian, M. S. (1987): Labor Market Areas for the United States. Washington, D. C. = Staff-Report No. AGES870721, Economic Research Service, US Department of Agriculture.

van Nuffel, N. (2007): Determination of the Number of Significant Flows in Origin - Destination Specific Analysis: The Case of Commuting in Flanders. In: Regional Studies 41, 4, 509-524.

van der Laan, L.; Schalke, R. (2001): Reality versus Policy: The Delineation and Testing of Local Labour Market and Spatial Policy Areas. In: European Planning Studies 11, 9, 201-221.

Wintzer, E. (2007): Regionalpolitik und New Economic Geography. Grundlagen, Modelle, Entwicklungen. Saarbrücken. 